\title{
HOMOGENEOUS DISTRIBUTIONS ON THE HEISENBERG GROUP AND REPRESENTATIONS OF $\mathrm{SU}(2,1)$
}

\author{
R. C. FABEC
}

\begin{abstract}
A 'Fourier' transform of tempered distributions on the Heisenberg group is defined to analyze homogeneous distributions relative the group of dilations $(z, t) \mapsto\left(r z, r^{2} t\right), r \in \mathbf{R}$. An inversion formula is derived for the abelian central Fourier transform of the distribution. These formulas are applied to the family of homogeneous distributions defining the intertwining operators for the group $\mathrm{SU}(2,1)$. Explicit unitary structures are determined on subquotient representations and their spectral decompositions on the minimal parabolic subgroup are obtained.
\end{abstract}

The object of this paper is two-fold. The first is to provide a framework in which to analyze the tempered distributions on the Heisenberg group $H$. Of particular use would be a transform defined in terms of the group structure on the space. This essentially is the content of the Plancherel Theorem when the distribution is defined by a Schwartz function or more generally by a square integrable function. Then the transform is a Hilbert-Schmidt operator valued function defined on the dual of the group. The Plancherel Theorem then provides an inversion process which allows one to reconstruct the function from this operator valued 'Fourier transform'. The transform is not unique but depends on the selection of representatives from the classes of irreducible unitary representations of $H$. In [2], L. Corwin and F. Greenleaf developed a method applicable to a class of nilpotent Lie groups of obtaining a smooth function as the 'Fourier transform' of a Schwartz function. This smooth transform depends on a smooth choice of irreducible representations and essentially provides a smooth parametrization of the kernels which define the operator valued Fourier transform.

In order to handle higher order distributions the transform must not be given by an operator valued function but by a distribution which is operator valued. In fact for a distribution $U$ we introduce a Fourier transform $\widehat{U}$ which is a distribution on a cosingular subset of the dual of $H$ and has values continuous linear transformations from the Schwartz space $\mathscr{S}(\mathbf{R})$ into the dual Schwartz space $\mathscr{S}^{*}(\mathbf{R})$. Moreover, there is an analog of the Plancherel theorem from which the distribution $U$ can be recovered from its Fourier transform $\widehat{U}$. This

Received by the editors September 22, 1989.

1980 Mathematics Subject Classification (1985 Revision). Primary 22E25; Secondary 22 E46.

Partially supported by LEQSF contract number 86-LBR-016-04. 
inversion process is not complete since some information about the distribution is lost by restricting test functions to only a part of the dual of $H$. The operator valued distribution $\widehat{U}$ can be shown to be equivalent to an infinite matrix of ordinary distributions. This matrix is obtained from a smooth parametrization of the matrix coefficients of the irreducible infinite-dimensional representations of $H$. When the transform is considered as an infinite-dimensional matrix, the transform of a convolution is an infinite matrix product which converges in the weak sense.

As in the case in analyzing homogeneous distributions on $\mathbf{R}$, the Fourier transform on $H$ provides the proper mechanism in which to analyze homogeneous distributions. The covariance of the transformed distribution $\widehat{U}$ provides sufficient information to determine each matrix element in the infinite transform up to a multiplicative constant.

In the second part of this paper these constants are determined for the family of homogeneous distributions invariant under rotation which define the KunzeStein, Knapp-Stein intertwining operators for the simple Lie group $\mathrm{SU}(2,1)$. The invariance under rotations makes the nondiagonal constants zero. The intertwining operators are convolution operators on $H$ defined by these distributions. Since in this case, the infinite matrices are diagonal, the realization of convolution as an infinite matrix product becomes a simple diagonal matrix product.

The convolution operators can then be used to obtain the complementary series and uniformly bounded representations as done in $[3,7,18]$, etc. This, however, is not our intent. Indeed, we use the intertwining operators at each point to obtain a nested sequence of invariant subspaces on whose quotients the intertwining operators and its principal values define invariant sesquilinear forms. When these forms are strictly definite, one obtains unitary subquotients of nonunitary principal series. This nested sequence of subspaces are the null spaces of a series of operators with smaller and smaller domains. Furthermore, by referring to the results of Kraljevic [9] one can see one obtains most of the nonunitary principal series and noncomplementary series dual.

Moreover, because the methods developed are analytic, it is possible to determine the spectral decomposition of the unitary subquotient representations on the minimal parabolic subgroup. This, as far as we are aware, cannot be obtained by algebraic methods.

Finally the author wishes to cite the paper [6] of Roger Howe. It was this paper which convinced the author that homogeneous distributions on the Heisenberg group could be understood in terms of an appropriate Heisenberg group theoretic 'Fourier transform'.

\section{Analysis on the Heisenberg Group}

In this part of the paper we develop methods to analyze distributions and convolutions by distributions on the Heisenberg group. Because of our interest 
in the group $\mathrm{SU}(2,1)$ and to keep notation simple we restrict ourselves to the 3-dimensional Heisenberg group $H=C \times \mathbf{R}$.

We begin by describing the Fock model. It is used to obtain the matrix coefficients for the infinite-dimensional irreducible unitary representations of $H$. These matrix coefficients are known to be Schwartz bases over $C$. The Plancherel Theorem is presented and the group theoretic transform $\widehat{U}$ is defined. $\widehat{U}$ is shown to completely determine $\widehat{U}$, the abelian Fourier transform over the center, on all of $C \times \mathbf{R}$ except on the singular set $C \times\{0\}$. Moreover a Plancherel formula relating $U$ and $\widehat{U}$ is presented. The distribution $\widehat{U}$ is shown to be determined by countably many distributions $\widehat{U}_{m, n}$.

Next we study convolution operators. Convolution operators by radial functions are shown to be infinite diagonal matrix operators relative to the 'Fourier transform' $U \mapsto \widehat{U}$. Finally, Sobolev spaces over $H$ are defined. Sufficient conditions insuring the boundedness of convolution operators between Sobolev spaces are obtained.

\section{THE FOCK MODEL}

The 3-dimensional Heisenberg group $H$ is the set $C \times \mathbf{R}$ with multiplication defined by $(z, t)(w, s)=(z+w, t+s+\sigma(z, w))$; where $\sigma$ is the symplectic form $\sigma\left(x+i y, x^{\prime}+i y^{\prime}\right)=y x^{\prime}-x y^{\prime}=\operatorname{Im}(z, w)$ and $(z, w)=z \bar{w}$.

Let $\mathscr{H}$ be the space of complex holomorphic functions $f$ on $\mathbf{C}$ such that

$$
\int|f(z)|^{2} e^{-|z|^{2}} d z<\infty
$$

Let $\langle$,$\rangle be the corresponding Hermitian product. Then for f$ in $\mathscr{H}$, one can show by using the residue theorem that

$$
f(0)=\frac{1}{\pi}\langle f, 1\rangle
$$

and in general

$$
f(z)=\frac{1}{\pi}\left\langle f, k_{z}\right\rangle, \quad \text { where } k_{z}(w)=e^{(w, z)} .
$$

$\mathscr{H}$ is the Fock space on $\mathscr{C}$ and is complete for (1.2) implies Cauchy sequences in $\mathscr{H}$ converge uniformly on bounded sets.

Define a representation $\pi$ of $H$ on $\mathscr{H}$ by

$$
\pi(z, t) f(w)=e^{-i t} e^{-|z|^{2} / 2} e^{(w, z)} f(w-z) .
$$

Theorem 1.1. $\pi$ is an irreducible unitary representation of $H$.

Proof. That $\pi$ is unitary and a representation is immediate. That $\pi$ is irreducible follows from the infinitesimal formulas (1.5) below.

The functions $z^{n}$ in $\mathscr{H}$ satisfy

$$
\left\langle z^{n}, z^{m}\right\rangle=\pi n ! \delta_{n, m},
$$


and thus $e_{n}=(\pi n !)^{-1 / 2} z^{n}, n=1,2, \ldots$, form a complete orthonormal basis of $\mathscr{H}$.

Let $X$ and $Y$ be the vectors in the Lie algebra of $H$ satisfying

$$
\exp (x X)=(x, 0), \quad \exp (y Y)=(i y, 0)
$$

Then one has

$$
\pi(X) z^{n}=z^{n+1}-n z^{n-1}, \quad \pi(Y) z^{n}=-i z^{n+1}-i n z^{n-1} .
$$

Matrix coefficients. Using (1.3) one obtains

$$
\begin{aligned}
\pi(w, 0) z^{n} & =e^{-|w|^{2} / 2} e^{(z, w)}(z-w)^{n} \\
& =e^{-|w|^{2} / 2} \sum_{k=0}^{\infty} \frac{z^{k} \bar{w}^{k}}{k !} \sum_{l=0}^{n}(-1)^{l}\left(\begin{array}{c}
n \\
l
\end{array}\right) w^{l} z^{n-l} \\
& =e^{-|w|^{2} / 2} \sum_{r=0}^{\infty} \sum_{l=\max (n-r, 0)}^{n}(-1)^{l}\left(\begin{array}{c}
n \\
l
\end{array}\right) \frac{1}{(l+r-n) !} w^{l} \bar{w}^{l+r-n} z^{r} \\
& =e^{-|w|^{2} / 2} \sum_{r=0}^{\infty} \bar{w}^{r-n} \sum_{l=\max (n-r, 0)}(-1)^{l}\left(\begin{array}{c}
n \\
l
\end{array}\right) \frac{|w|^{2 l}}{(l+r-n) !} z^{r} .
\end{aligned}
$$

Hence one has the following.

Proposition 1.2.

$$
\left\langle\pi(w, 0) e_{n}, e_{r}\right\rangle=\left(\frac{r !}{n !}\right)^{1 / 2} e^{-|w|^{2} / 2} \bar{w}^{r-n} \sum_{l=\max (n-r, 0)}^{n}(-1)^{l}\left(\begin{array}{l}
n \\
l
\end{array}\right) \frac{|w|^{2 l}}{(l+r-n) !}
$$

The Laguerre polynomials are defined by

$$
L_{n}^{\alpha}(x)=\sum_{m=0}^{n}(-1)^{m} \frac{\Gamma(n+\alpha+1)}{(n-m) ! \Gamma(\alpha+m+1)} \frac{x^{m}}{m !} .
$$

Hence since $\left\langle\pi(w, 0) e_{n}, e_{r}\right\rangle=\overline{\left\langle\pi(-w, 0) e_{r}, e_{n}\right\rangle}$, one has

$$
\left\langle\pi(w, 0) e_{n}, e_{r}\right\rangle=\left(\frac{n !}{r !}\right)^{1 / 2} e^{-|w|^{2} / 2} \bar{w}^{r-n} L_{n}^{r-n}\left(|w|^{2}\right) \quad \text { if } r \geq n
$$

and

$$
\left\langle\pi(w, 0) e_{n}, e_{r}\right\rangle=(-1)^{n-r}\left(\frac{r !}{n !}\right)^{1 / 2} e^{-|w|^{2} / 2} w^{n-r} L_{r}^{n-r}\left(|w|^{2}\right) \text { if } n>r .
$$

It follows from (1.6) and (1.7) that the representation $\pi$ is square integrable modulo the center.

Let $\overline{\mathscr{H}}$ be the conjugate space to $\mathscr{H}$. Thus $\overline{\mathscr{H}}=\mathscr{H},\langle f, g\rangle^{-}=\overline{\langle f, g\rangle}$, and $\lambda f=\bar{\lambda} f$. Define $\pi^{\lambda}$ on $\mathscr{H}$ by

$$
\pi^{\lambda}(z, t)=\pi(\sqrt{\lambda} z, \lambda t) \text { for } \lambda>0 .
$$


For $\lambda<0$, set $\pi^{\lambda}=\overline{\pi^{-\lambda}}$. Thus $\pi^{\lambda}(0, t)=e^{-i \lambda t}$ and by the Stone-Von Neumann Theorem, $\pi^{\lambda}$ is the unique up to equivalence irreducible unitary representation of $\mathscr{H}$ with central character $e^{-i \lambda t}$. One has the following matrix coefficients:

$$
\begin{aligned}
\left\langle\pi^{\lambda}(w, 0) e_{n}, e_{r}\right\rangle=\left(\frac{n !}{r !}\right)^{1 / 2} e^{-\lambda|w|^{2} / 2} \lambda^{(r-n) / 2} \bar{w}^{r-n} L_{n}^{r-n}\left(\lambda|w|^{2}\right) & \\
& \text { if } \lambda>0, r \geq n
\end{aligned}
$$

and

$$
\begin{array}{r}
\left\langle\pi^{\lambda}(w, 0) e_{n}, e_{r}\right\rangle=(-1)^{n-r}\left(\frac{r !}{n !}\right)^{1 / 2} e^{-\lambda|w|^{2} / 2} \lambda^{(n-r) / 2} w^{n-r} L_{r}^{n-r}\left(\lambda|w|^{2}\right), \\
\text { if } \lambda>0, n \geq r .
\end{array}
$$

If $\lambda<0$, the matrix coefficients of $\pi^{\lambda}$ are the conjugates to these.

\section{The Plancherel Theorem}

Let $\mathscr{S}(\mathbf{R})$ be Schwartz space on $\mathbf{R}$. Recall one has

$$
\iint f(x) e^{-i x y} e^{i y z} d x d y=2 \pi f(z),
$$

for $f \in \mathscr{S}(\mathbf{R})$.

For each real number $\lambda$ define an irreducible unitary representation $\pi_{\lambda}$ of $H$ on $L^{2}(\mathbf{R})$ by

$$
\begin{aligned}
\pi_{\lambda}(x, 0) f(r) & =f(r-x), \\
\pi_{\lambda}(i y, 0) f(r) & =e^{-2 i \lambda y r} f(r), \\
\pi_{\lambda}(0, t) f(r) & =e^{-i \lambda t} f(r) .
\end{aligned}
$$

The Stone-Von Neumann Theorem implies $\pi_{\lambda}$ is unitarily equivalent to $\pi^{\lambda}$ for $\lambda \neq 0$.

Clearly,

$$
\pi_{\lambda}(x+i y, t)=\pi_{1}(x+i \lambda y, \lambda t) .
$$

Theorem 2.1.

$$
\int_{\mathbf{C}}\left\langle v, \pi_{\lambda}(z, 0) w\right\rangle \overline{\left\langle v^{\prime}, \pi_{\lambda}(z, 0) w^{\prime}\right\rangle} d z=\frac{\pi}{|\lambda|}\left\langle v, v^{\prime}\right\rangle\left\langle w^{\prime}, w\right\rangle,
$$

for $v, v^{\prime}, w, w^{\prime} \in \mathscr{S}(\mathbf{R})$.

Proof. This follows from an application of (2.1).

Let $\mathscr{S}(H)$ be the space of Schwartz functions on $H$. For $f \in \mathscr{S}(H)$ and $\sigma$ a unitary representation of $\mathscr{H}$ define

$$
\sigma(f)=\int f(h) \sigma(h) d h .
$$


Then the operator $\pi_{\lambda}(f)$ is a Hilbert-Schmidt operator having Schwartz kernel

$$
P_{\lambda}(f)(x, y)=\iint f(x-y+i r, t) e^{-i \lambda t} e^{-i \lambda r(x+y)} d r d t
$$

Thus

$$
\pi_{\lambda}(f) \phi(x)=\int P_{\lambda}(f)(x, y) \phi(y) d y .
$$

In particular, $\pi_{\lambda}(f)$ is trace class. For $f \in \mathscr{S}(H)$, we shall use $\hat{f}$ to denote the Fourier transform of $f$ over the center of $H$. Hence

$$
\hat{f}(z, \lambda)=\int f(z, t) e^{-i \lambda t} d t
$$

Theorem 2.2. $\operatorname{tr}\left(\pi_{\lambda}(f)\right)=\frac{\pi}{|\lambda|} \hat{f}(0, \lambda)$.

Proof.

$$
\begin{aligned}
\operatorname{tr}\left(\pi_{\lambda}(f)\right) & =\int P_{\lambda} f(x, x) d x=\iiint f(i r, t) e^{-i \lambda t} e^{-2 \lambda i r x} d r d t d x \\
& =\frac{1}{2|\lambda|} \iiint f(i r, t) e^{-i r x} d r d x e^{-i \lambda t} d t=\frac{\pi}{|\lambda|} \hat{f}(0, \lambda)
\end{aligned}
$$

An immediate consequence of Theorem 2.2 is the Plancherel Theorem on $H$. If $T$ is a Hilbert-Schmidt operator, then $\|T\|_{2}=\operatorname{tr}\left(T^{*} T\right)^{1 / 2}$ is the HilbertSchmidt norm of $T$.

Theorem 2.3. Assume $f$ is a Schwartz function on $H$. Then:

(1) $f(e)=\left(1 / 2 \pi^{2}\right) \int \operatorname{tr}\left(\pi_{\lambda}(f)\right)|\lambda| d \lambda$,

(2) $\|f\|_{2}^{2}=\left(1 / 2 \pi^{2}\right) \int\left\|\pi_{\lambda}(f)\right\|_{2}^{2}|\lambda| d \lambda$,

(3) $f(h)=\left(1 / 2 \pi^{2}\right) \int \operatorname{tr}\left(\pi_{\lambda}\left(h^{-1}\right) \pi_{\lambda}(f)\right)|\lambda| d \lambda$.

Proof. First note by $(2.1)$

$$
\frac{1}{2 \pi^{2}} \int \operatorname{tr}\left(\pi_{\lambda}(f)\right)|\lambda| d \lambda=\frac{1}{2 \pi} \int \hat{f}(0, \lambda) d \lambda=f(0,0) .
$$

Thus (1) holds. Using $f * f^{*}(0)=\|f\|_{2}^{2}$ and $\pi_{\lambda}(f(h \cdot))=\pi_{\lambda}\left(h^{-1}\right) \pi_{\lambda}(f)$, it follows that (2) and (3) hold.

The usefulness of Theorem 2.3 is in part dependent on the fact that each $\pi_{\lambda}$ can be replaced by any unitarily equivalent representation $\sigma_{\lambda}$.

Corollary 2.4. The mapping $f \mapsto U(f)$, where $U(f)(\lambda)=\pi_{\lambda}(f)$, is a unitary isomorphism of $L^{2}(H)$ onto $\int^{\oplus} \mathbf{H S}\left(L^{2}(\mathbf{R})\right)|\lambda| d \lambda$. Moreover, if $B$ is the biregular representation of $H$ on $L^{2}(H)$ defined by

$$
B\left(g_{1}, g_{2}\right) f(h)=f\left(g_{1}^{-1} h g_{2}\right)
$$

then

$$
U\left(B\left(g_{1}, g_{2}\right) f\right)(\lambda)=\pi_{\lambda}\left(g_{1}\right) U(f) \pi_{\lambda}\left(g_{2}^{-1}\right)
$$


We end this section by noting that the matrix coefficients of the representation $\pi^{\lambda}$ for $\lambda \neq 0$ form a complete orthonormal basis of $L^{2}(\mathbf{C})$.

In fact by Theorem 2.1 one has

$$
\int_{\mathbf{C}}\left\langle\pi_{\lambda}(w, 0) e_{m}, e_{n}\right\rangle \overline{\left\langle\pi_{\lambda}(w, 0) e_{r}, e_{s}\right\rangle} d w=\frac{\pi}{|\lambda|} \delta_{m, r} \delta_{n, s} .
$$

Thus the matrix coefficients

$$
(|\lambda| / \pi)^{1 / 2}\left\langle\pi^{\lambda}(w, 0) e_{m}, e_{n}\right\rangle
$$

form an orthonormal set. Moreover, by Proposition 1.2, the linear span of the functions $\left\langle\pi(\sqrt{\lambda} w, 0) e_{m}, e_{n}\right\rangle$ contains all functions of form $P(w, \bar{w}) e^{-\lambda|w|^{2} / 2}$ where $P$ is a polynomial in $w$ and $\bar{w}$ for $\lambda>0$.

Theorem 2.5. For $\lambda \neq 0$ the Schwartz functions $(|\lambda| / \pi)^{1 / 2}\left\langle\pi^{\lambda}(w, 0) e_{m}, e_{n}\right\rangle$ form a complete orthonormal basis of $L^{2}(\mathbf{C})$.

\section{Decomposition of Schwartz functions}

In [6] Roger Howe discusses a strong Stone-Von Neumann Theorem. We shall make extensive use of this theorem. We discuss this theorem and use it to obtain Schwartz space decompositions of smooth functions. Let $\rho$ be the unitary representation $\pi_{1}$.

For a Schwartz function $f$ on $\mathbf{C}$ set

$$
\rho(f)=\int f(z) \rho(z, 0) d z
$$

Then $\rho(f)$ is an operator on $L^{2}(\mathbf{R})$ given by the Schwartz kernel

$$
K_{f}(x, y)=\int f(x-y+i r) e^{-i r(x+y)} d r .
$$

We will identify functions on $\mathbf{R}^{2}$ and on $\mathbf{C}$. In particular, by $K_{f}(x+i y)$ we shall mean $K_{f}(x, y)$.

Theorem 3.1 (Strong Stone-Von Neumann Theorem). (1) $\|f\|^{2}=\frac{1}{\pi}\left\|K_{f}\right\|^{2}$.

(2) $f \mapsto K_{f}$ is a topological isomorphism of $\mathscr{S}(\mathbf{C})$ onto $\mathscr{S}(\mathbf{C})$.

(3) If $f(z)=\langle\rho(z, 0) h, k\rangle$, then $K_{f}(x, y)=\pi h(-x) \overline{k(-y)}$.

(4) If $f(z)=\langle h, \rho(z, 0) k\rangle$, then $K_{f}(x, y)=\pi h(x) \overline{k(y)}$.

(5) $K_{R_{h_{1}} L_{h_{2}} f}(x, y)=(\rho \otimes \bar{\rho})\left(h_{1}, h_{2}\right) K_{f}(x, y)$ where

$$
R_{(w, t)} f(z)=e^{i(t+\sigma(z, w))} f(z+w), \quad L_{(w, t)} f(z)=e^{-i(t+\sigma(w, z))} f(z-w) .
$$

The proof is essentially an application of Theorem 2.1.

For the general central character case we set

$$
\rho_{\lambda}(f)=\int f(z) \pi_{\lambda}(z, 0) d z .
$$


Since $\pi_{\lambda}(x+i y, 0)=\pi_{1}(x+i \lambda y, 0)$, the operator $\rho_{\lambda}(f)$ has kernel $K_{f}^{\lambda}$ defined by

$$
K_{f}^{\lambda}=\frac{1}{|\lambda|} K_{f(x+i y / \lambda)}
$$

Thus

$$
\begin{gathered}
\|f\|_{2}^{2}=\frac{|\lambda|}{\pi}\left\|K_{f}^{\lambda}\right\|^{2}, \\
K_{\left\langle\pi_{\lambda}(z, 0) h, k\right\rangle}^{\lambda}(x, y)=\frac{\pi}{|\lambda|} h(-x) \overline{k(-y)}, \\
K_{\left\langle h, \pi_{\lambda}(z, 0) k\right\rangle}^{\lambda}(x, y)=\frac{\pi}{|\lambda|} h(x) \overline{k(y)},
\end{gathered}
$$

and if $R^{\lambda}(x+i y, t)=R(x+i \lambda y, \lambda t)$ and $L^{\lambda}(x+i y, t)=L(x+i \lambda y, \lambda t)$, then

$$
K_{R_{h_{1}}^{\lambda} L_{h_{2}}^{\lambda} f}^{\lambda}=\left(\pi_{\lambda} \otimes \pi_{-\lambda}\right)\left(h_{1}, h_{2}\right) K_{f}^{\lambda}
$$

The relationship between $K^{\lambda}$ and $P_{\lambda}$ defined in (2.4) is

$$
P_{\lambda}(f)=K_{\hat{f}(\cdot, \lambda)}^{\lambda} \cdot
$$

We thus define a mapping on $\mathscr{S}(H)$ by

$$
K f(z, \lambda)=K_{f(\cdot, \lambda)}^{\lambda}(z) .
$$

By 3.4 one sees

$$
\begin{aligned}
K f(u+i v, \lambda) & =\frac{1}{|\lambda|} K_{f(x+i y / \lambda, \lambda)}(u+i v) \\
& =\frac{1}{|\lambda|} \int f(u-v+i r / \lambda, \lambda) e^{-i r(u+v)} d r \\
& =\int f(u-v+i r, \lambda) e^{-i r \lambda(u+v)} d r
\end{aligned}
$$

Thus

$$
K f(u+i v, \lambda)=F_{2} f(u-v, \lambda(u+v), \lambda),
$$

where $F_{2}: \mathscr{S}(H) \rightarrow \mathscr{S}(H)$ is the partial Fourier transform defined by

$$
\left(F_{2} f\right)(x+i y, \lambda)=\int f(x+i r, \lambda) e^{-i r y} d r .
$$

Define $T$ on $H$ by

$$
T(x+i y, \lambda)=(x-y+i \lambda(x+y), \lambda) .
$$

Then

$$
K(f)=F_{2}(f) \circ T
$$


Note that $T$ is $C^{\infty}$ on $H$ but is not invertible. However, $T$ is a $C^{\infty}$ diffeomorphism of the open submanifold $H_{0}=H-(\mathbf{C} \times\{0\})$. Its inverse is given by

$$
T^{-1}(x+i y, \lambda)=(1 / 2(x+y / \lambda+i(-x+y / \lambda)), \lambda) .
$$

We let $\mathscr{S}_{0}(H)$ denote the space of Schwartz functions on $H$ vanishing for $\lambda$ near 0 . A sequence $f_{n}$ is defined to converge in $\mathscr{S}_{0}(H)$ if it converges in the Schwartz topology and all the $f_{n}$ vanish together for $\lambda$ near 0 . We then have:

Proposition 3.2. $K$ is a topological isomorphism of $\mathscr{S}_{0}(H)$.

We shall apply this proposition to decompose Schwartz functions in $\mathscr{S}_{0}(H)$. First we take a Schwartz basis $e_{m}$ of orthonormal functions in $L^{2}(\mathbf{R})$. Thus each $e_{m}$ is a Schwartz function, the functions $e_{m}$ are orthonormal in $L^{2}(\mathbf{R})$ and if $f=\sum a_{m} e_{m}$, then $f$ is Schwartz iff $\sum\left(1+m^{k}\right)\left|a_{m}\right|<\infty$ for each natural number $k$. We remark that the $e_{m}$ 's defined after (1.4) correspond to the Hermite functions in $L^{2}(\mathbf{R})$ and thus may be considered such a basis.

The discussion to be held below using the representations $\pi_{\lambda}$ and a Schwartz basis $e_{m}$ for $L^{2}(\mathbf{R})$ also applies to the representations $\pi^{\lambda}$ on Fock space and the basis $e_{m}$ of $\mathscr{H}$ defined after Theorem 1.1. A direct argument may be given. There are alterations in the kernel operators $K_{\lambda}$ and in the transformation $K$.

Define

$$
h_{m, n}(z, \lambda)=\left\langle\pi_{\lambda}(z, 0) e_{m}, e_{n}\right\rangle
$$

Then by 3.7

$$
K_{\bar{h}_{m, n}}^{\lambda}(x+i y)=\frac{\pi}{|\lambda|} e_{n}(x) \bar{e}_{m}(y),
$$

and thus by 3.5 the functions $(|\lambda| / \pi)^{1 / 2} \bar{h}_{m, n}(z, \lambda)$ form an orthonormal basis of $\mathscr{S}(\mathbf{C})$ whenever $\lambda \neq 0$. Thus if $f \in L^{2}(H)$ one has

$$
f(z, \lambda)=\sum c_{m, n}(\lambda) \bar{h}_{m, n}(z, \lambda)
$$

where $c_{m, n}(\lambda)=(|\lambda| / \pi) \int f(z, \lambda) h_{m, n}(z, \lambda) d z$. Moreover, $f(\cdot, \lambda) \in \mathscr{S}(\mathbf{C})$ iff $\sum(1+m+n)^{k}\left|c_{m, n}(\lambda)\right|<\infty$ for each $k$.

For $f \in \mathscr{S}(H)$, define

$$
\widehat{f}_{m, n}(\lambda)=\left\langle\pi_{\lambda}(f) e_{m}, e_{n}\right\rangle .
$$

Then

$$
\begin{aligned}
\hat{f}_{m, n}(\lambda) & =\iint f(w, t)\left\langle\pi_{\lambda}(w, t) e_{m}, e_{n}\right\rangle d t d w \\
& =\iint f(w, t) e^{-i \lambda t} h_{m, n}(w, \lambda) d t d w \\
& =\int \hat{f}(w, \lambda) h_{m, n}(w, \lambda) d w .
\end{aligned}
$$


Theorem 3.2. Suppose $f$ is a Schwartz function on $H$ whose central Fourier transform $\hat{f}$ is in $\mathscr{S}_{0}(H)$. Then

$$
\hat{f}(z, \lambda)=\frac{|\lambda|}{\pi} \sum_{m, n} \hat{f}_{m, n}(\lambda) \bar{h}_{m, n}(z, \lambda),
$$

and this sum converges in $\mathscr{S}_{0}(H)$.

Proof. By Proposition 3.2, it suffices to show

$$
K \hat{f}=\sum_{m, n} K\left(\frac{|\lambda|}{\pi} \widehat{f}_{m, n}(\lambda) \bar{h}_{m, n}(z, \lambda)\right)
$$

in $\mathscr{S}_{0}(H)$. But by $(3.15)$

$$
\begin{aligned}
K\left(\widehat{f}_{m, n}(\lambda) \bar{h}_{m, n}(z, \lambda)\right) & =\widehat{f}_{m, n}(\lambda) K_{\bar{h}_{m, n}^{\lambda}}(x+i y) \\
& =\widehat{f}_{m, n}(\lambda) \frac{\pi}{|\lambda|} e_{n}(x) \bar{e}_{m}(y) .
\end{aligned}
$$

Hence

$$
\sum_{m, n} K\left(\frac{|\lambda|}{\pi} \widehat{f}_{m, n}(\lambda) \bar{h}_{m, n}(z, \lambda)\right)=\sum_{m, n} \widehat{f}_{m, n}(\lambda) e_{n}(x) \bar{e}_{m}(y) .
$$

The proof will be complete if

$$
\left\langle K \hat{f}(\cdot, \lambda), e_{n} \otimes \bar{e}_{m}\right\rangle=\hat{f}_{m, n}(\lambda) .
$$

But

$$
\begin{aligned}
\hat{f}_{m, n}(\lambda) & =\left\langle\pi_{\lambda}(f) e_{m}, e_{n}\right\rangle=\int \hat{f}(z, \lambda)\left\langle\pi_{\lambda}(z, 0) e_{m}, e_{n}\right\rangle d z \\
& =\left\langle\rho_{\lambda}(\hat{f}(\cdot, \lambda)) e_{m}, e_{n}\right\rangle=\int K_{\hat{f}(\cdot, \lambda)}^{\lambda}(x+i y) e_{m}(y) \bar{e}_{n}(x) d y d x \\
& =\int K \hat{f}(x+i y, \lambda) \overline{e_{n}(x) \bar{e}_{m}(y)} d x d y \\
& =\left\langle K \hat{f}(\cdot, \lambda), e_{n}(x) \otimes \bar{e}_{m}\right\rangle .
\end{aligned}
$$

\section{The Plancherel Theorem for distributions}

Let $\mathscr{S}^{*}(H)$ be the space of tempered distributions on $H$ and let $\mathscr{S}_{0}(\mathbf{R})$ denote the space of Schwartz functions on $\mathbf{R}$ vanishing near 0 . Let $\mathscr{L}\left(\mathscr{S}(\mathbf{R}), \mathscr{S}^{*}(\mathbf{R})\right)$ be the space of continuous linear transformations from $\mathscr{S}(\mathbf{R})$ into $\mathscr{S}^{*}(\mathbf{R})$, where $\mathscr{S}^{*}(\mathbf{R})$ has the weak* topology. $\mathscr{L}\left(\mathscr{S}(\mathbf{R}), \mathscr{S}^{*}(\mathbf{R})\right)$ is given the simple topology.

Let $U \in \mathscr{S}^{*}(H)$. We define a 'distribution' on $\mathscr{S}_{0}(\mathbf{R})$ with values in $\mathscr{L}\left(\mathscr{S}(\mathbf{R}), \mathscr{S}^{*}(\mathbf{R})\right)$ formally by

$$
\widehat{U}(\phi)=\int d U(z, t) \int \phi(\lambda) \pi_{\lambda}(z, t) d \lambda .
$$


More specifically, if $f$ and $h$ are in $\mathscr{S}(\mathbf{R})$, then

$$
\widehat{U}(\phi)(f)(h)=\int d U(z, t) \int \phi(\lambda)\left\langle\pi_{\lambda}(z, t) f, \bar{h}\right\rangle d \lambda
$$

Theorem 4.1. $\widehat{U}$ is a continuous linear map from the space $\mathscr{S}_{0}(\mathbf{R})$ into the space $\mathscr{L}\left(\mathscr{S}(\mathbf{R}), \mathscr{S}^{*}(\mathbf{R})\right)$.

Proof. Note by Theorem 3.1 that

$$
\phi(\lambda)\left\langle\pi_{\lambda}(z, 0) f, \bar{h}\right\rangle \in \mathscr{S}_{0}(\mathbf{R}) \quad \text { if } \phi \in \mathscr{S}_{0}(\mathbf{R}) \text { and } f, h \in \mathscr{S}(\mathbf{R}) .
$$

Moreover, one sees by (3.6) that $\phi(\lambda)\left\langle\pi_{\lambda}(z, 0) f, \bar{h}\right\rangle$ is continuous into $\mathscr{S}(H)$ as a mapping on $\mathscr{S}_{0}(\mathbf{R}) \times \mathscr{S}(\mathbf{R}) \times \mathscr{S}(\mathbf{R})$. Since $\pi_{\lambda}(z, t)=e^{-i \lambda t} \pi_{\lambda}(z, 0)$, one has $\widehat{U}(\phi)(f)(h)=U\left(\left(\phi(\lambda)\left\langle\pi_{\lambda}(z, 0) f, \bar{h}\right\rangle\right) \hat{)}\right)$ and the result follows.

In the case where the distribution $U$ is given by a Schwartz function $f$, the distribution $\widehat{f}$ is given by an operator valued function. Indeed

$$
\begin{aligned}
\hat{f}(\phi) & =\iint f(z, t) \int \phi(\lambda) \pi_{\lambda}(z, t) d \lambda d z d t \\
& =\int \phi(\lambda) \iint f(z, t) \pi_{\lambda}(z, t) d z d t=\int \phi(\lambda) \pi_{\lambda}(f) d \lambda .
\end{aligned}
$$

Thus the distribution $f$ is defined by the operator valued function $\lambda \mapsto \pi_{\lambda}(f)$ which is the analog of a Fourier transform of $f$. In this case we shall write

$$
\widehat{f}(\lambda)=\pi_{\lambda}(f)
$$

Moreover, since $\pi_{\lambda}(f)=\int f(z, t) e^{-i \lambda t} \pi_{\lambda}(z, 0) d z d t$, we see that

$$
\pi_{\lambda}(f)=\int \hat{f}(z, \lambda) \pi_{\lambda}(z, 0) d z
$$

and thus $\hat{f}(\lambda)$ equals $\rho_{\lambda}(\hat{f}(\cdot, \lambda))$.

Furthermore, the operator $\hat{f}(\phi)$ has a Schwartz kernel. Indeed, by (3.11) the kernel is given by

$$
\begin{aligned}
K_{\widehat{f}_{(\phi)}}(x, y) & =\int \phi(\lambda) K_{\hat{f}(\cdot, \lambda)}^{\lambda}(x+i y) d \lambda \\
& =\int \phi(\lambda) F_{2} \hat{f}(x-y, \lambda(x+y), \lambda) d \lambda .
\end{aligned}
$$

In particular, $f(\phi)$ is a continuous linear transformation of $\mathscr{S}(\mathbf{R})$ when $\phi$ is in $\mathscr{S}_{0}(\mathbf{R})$.

We next attempt to reconstruct the distribution $U$ from its 'Fourier transform' $\widehat{U}$. First we note that if $f$ is a Schwartz function on $H$, then

$$
f(z, t)=\frac{1}{2 \pi^{2}} \int \operatorname{tr}\left(\pi_{\lambda}(-z,-t) \pi_{\lambda}(f)\right)|\lambda| d \lambda .
$$


Thus formally,

$$
\begin{aligned}
\int d U(z, t) f(z, t) & =\frac{1}{2 \pi^{2}} \int d U(z, t) \operatorname{tr}\left(\pi_{\lambda}(-z,-t) \pi_{\lambda}(f)\right)|\lambda| d \lambda \\
& =\frac{1}{2 \pi^{2}} \operatorname{tr}\left(\int d U(-z,-t) \int \pi_{\lambda}(z, t)|\lambda| \pi_{\lambda}(f) d \lambda\right) \\
& =\frac{1}{2 \pi^{2}} \operatorname{tr} \int d \check{U}(\lambda)\left(|\lambda| \pi_{\lambda}(f)\right),
\end{aligned}
$$

where $d \check{U}(z, t)=d U(-z,-t)$. We shall show this formula holds when a suitable interpretation is made for the trace and when $\hat{f}(z, \lambda)$ vanishes for $\lambda$ near 0 .

First we extend the domain of the distribution $\widehat{U}$. Indeed, suppose $\phi \epsilon$ $\mathscr{S}_{0}(\mathbf{R}, \mathscr{L}(\mathscr{S}(\mathbf{R}), \mathscr{S}(\mathbf{R})))$. Define $\widehat{U}(\phi)$ by

$$
\widehat{U}(\phi)(f)(h)=\int d U(z, t) \int\left\langle\pi_{\lambda}(z, t) \phi(\lambda) f, \bar{h}\right\rangle d \lambda
$$

By an argument similar to that given for Theorem $3.1, \widehat{U}$ is a distribution on $\mathscr{S}_{0}(\mathbf{R}, \mathscr{L}(\mathscr{S}(\mathbf{R}), \mathscr{S}(\mathbf{R})))$ with values in $\mathscr{L}\left(\mathscr{S}(\mathbf{R}), \mathscr{S}^{*}(\mathbf{R})\right)$. We denote this operator by $\int d \hat{U}(\lambda) \phi(\lambda)$ and define for a Schwartz basis $\left\{e_{m}\right\}$ of $\mathscr{S}(\mathbf{R})$ a trace by

$$
\operatorname{tr}\left(\int d \widehat{U}(\lambda) \phi(\lambda)\right)=\sum_{m}\left(\int d \widehat{U}(\lambda) \phi(\lambda) e_{m}, \bar{e}_{m}\right)
$$

where $(T, f)=T(f)$ for $T \in \mathscr{S}^{*}(\mathbf{R})$ and $f \in \mathscr{S}(\mathbf{R})$.

Our main result concerning the distribution $\widehat{U}$ allows one to reconstruct the distribution $\widehat{U}$ on a dense open submanifold.

Theorem 4.2 (The Plancherel Theorem for distributions). Suppose $U$ is a tempered distribution on $H$. Then whenever $\hat{f} \in \mathscr{S}_{0}(\mathbf{R})$, one has

$$
\check{U}(f)=\frac{1}{2 \pi^{2}} \operatorname{tr}\left(\int d \widehat{U}(\lambda)|\lambda| \pi_{\lambda}(f)\right) .
$$


Proof.

$$
\begin{aligned}
\frac{1}{2 \pi^{2}} \sum_{m}\left(\int d \widehat{U}(\lambda)|\lambda| \pi_{\lambda}(f) e_{m}, \bar{e}_{m}\right) \\
=\frac{1}{2 \pi^{2}} \sum \int d U(z, t) \int|\lambda|\left\langle\pi_{\lambda}(z, t) \pi_{\lambda}(f) e_{m}, e_{m}\right\rangle d \lambda \\
=\frac{1}{2 \pi^{2}} \sum \int d U(z, t) \iint|\lambda| e^{-i \lambda t} \hat{f}(w, \lambda) \\
\quad \times\left\langle\pi_{\lambda}(w, 0) e_{m}, \pi_{\lambda}(-z, 0) e_{m}\right\rangle d w d \lambda \\
=\frac{1}{2 \pi^{2}} \sum_{m} \int d U(z, t) \iint|\lambda| e^{-i \lambda t} \hat{f}(w, \lambda) \\
\quad \times \sum_{k}\left\langle\pi_{\lambda}(w, 0) e_{m}, e_{k}\right\rangle\left\langle e_{k}, \pi_{\lambda}(-z, 0) e_{m}\right\rangle d w d \lambda \\
=\frac{1}{2 \pi^{2}} \sum_{m} \int d U(z, t) \iint|\lambda| e^{-i \lambda t} \sum_{k} \hat{f}_{m, k}(\lambda) \bar{h}_{m, k}(-z, \lambda) d \lambda
\end{aligned}
$$

But by Theorem $3.2 \sum_{m} \sum_{k}|\lambda| \hat{f}_{m, k}(\lambda) \bar{h}_{m, k}(-z, \lambda)$ converges in $\mathscr{S}_{0}(H)$ to $\pi \hat{f}(-z, \lambda)$. Hence by $(2.1)$

$$
\begin{aligned}
\frac{1}{2 \pi^{2}} \operatorname{tr}\left(\int d \widehat{U}(\lambda)|\lambda| \pi_{\lambda}(f)\right) & =\frac{1}{2 \pi} \int d U(z, t) \int e^{-i \lambda t} \hat{f}(-z, \lambda) d \lambda \\
& =\int d U(z, t) f(-z,-t) .
\end{aligned}
$$

We note this theorem holds for any choice of Schwartz basis. Moreover, it remains valid if the irreducible unitary representations $\pi_{\lambda}$ on $L^{2}(\mathbf{R})$ are replaced by the equivalent representations $\pi^{\lambda}$ on Fock space and one uses the orthonormal basis of Fock space defined in $\S 1$.

\section{Distributions AND CONVOLUTIONS}

The Plancherel Theorem for distributions shows that the central Fourier transform of a tempered distribution $U$ on the Heisenberg group is determined on a cosingular subset by the distribution $\overparen{U}$. But for any Schwartz basis $e_{m}$ of $\mathscr{S}(\mathbf{R})$, the distribution $\widehat{U}$ is determined by the family of distributions $\widehat{U}_{m, n}$ defined on $\mathscr{S}_{0}(\mathbf{R})$ by:

$$
\widehat{U}_{m, n}(\phi)=\left(\widehat{U}(\phi) e_{m}, \bar{e}_{n}\right)
$$

Thus

$$
\begin{aligned}
\widehat{U}_{m, n}(\phi) & =\int d U(z, t) \int \phi(\lambda)\left\langle\pi_{\lambda}(z, t) e_{m}, e_{n}\right\rangle d \lambda \\
& =\int d U(z, t) \int \phi(\lambda) h_{m, n}(z, \lambda) e^{-i \lambda t} d \lambda \\
& =\widehat{U}\left(\phi(\lambda) h_{m, n}(z, \lambda)\right),
\end{aligned}
$$

where $\widehat{U}(f)=U(\hat{f})$. 
Next suppose $\hat{f} \in \mathscr{S}_{0}(H)$. Then

$$
\begin{aligned}
& \left(\int d \widehat{U}(\lambda)|\lambda| \pi_{\lambda}(f) e_{m}, \bar{e}_{m}\right)=\int d U(z, t) \int\left\langle|\lambda| \pi_{\lambda}(z, t) \pi_{\lambda}(f) e_{m}, e_{m}\right\rangle d \lambda \\
& =\int d U(z, t) \int e^{-i \lambda t}|\lambda|\left\langle\pi_{\lambda}(f) e_{m}, \pi_{\lambda}(-z, 0) e_{m}\right\rangle d \lambda \\
& =\int d U(z, t) \int e^{-i \lambda t} \sum_{k}|\lambda|\left\langle\pi_{\lambda}(f) e_{m}, e_{k}\right\rangle\left\langle e_{k}, \pi_{\lambda}(-z, 0) e_{m}\right\rangle d \lambda \\
& =\sum_{k} \int d U(z, t) \int e^{-i \lambda t}|\lambda| \widehat{f}_{m, k}(\lambda) h_{k, m}(z, \lambda) d \lambda \\
& =\sum_{k} \widehat{U}_{k, m}\left(|\lambda| \hat{f}_{m, k}(\lambda)\right),
\end{aligned}
$$

where the change in summation follows from Theorem 3.2. We thus see Theorem 5.1. Suppose the central Fourier transform $\hat{f}$ is in $\mathscr{S}_{0}(H)$. Then

$$
\check{U}(f)=\frac{1}{2 \pi^{2}} \sum_{k, m} \widehat{U}_{k, m}\left(|\lambda| \widehat{f}_{m, k}(\lambda)\right) .
$$

Theorem 5.1 shows how to reconstruct the distribution $\widehat{U}$ off the singular set $\mathbf{C} \times\{0\}$ from the distributions $\widehat{U}_{m, n}$. For distributions that are $L^{1}$ or $L^{2}$ functions, the process is complete.

Theorem 5.2. Let $U=u_{1}+u_{2}$ where $u_{1} \in L^{1}(H)$ and $u_{2} \in L^{2}(H)$. Then

$$
\check{U}(f)=\frac{1}{2 \pi^{2}} \sum_{k, m} \int \widehat{U}_{k, m}(\lambda) \widehat{f}_{m, k}(\lambda)|\lambda| d \lambda,
$$

for all Schwartz functions $f$.

Proof. By the Plancherel Theorem, $\pi_{\lambda}(f)$ is trace class for all $\lambda \neq 0$ and $\pi_{\lambda}\left(u_{2}\right)$ is a Hilbert-Schmidt operator for a.e. $\lambda$. Moreover, $\left\|\pi_{\lambda}\left(u_{1}\right)\right\| \leq\left\|u_{1}\right\|_{1}$. Thus $\pi_{\lambda}(f) \pi_{\lambda}(U)$ is trace class a.e. $\lambda$. Furthermore, note that

$$
\left|\lambda \operatorname{tr}\left(\pi_{\lambda}(f) \pi_{\lambda}\left(u_{1}\right)\right)\right| \leq|\lambda| \operatorname{tr}\left(\pi_{\lambda}\left(f * f^{*}\right)\right)^{1 / 2}\left\|u_{1}\right\|_{1}
$$

is integrable in $\lambda$ and by Corollary 2.4

$$
\left|\lambda \operatorname{tr}\left(\pi_{\lambda}(f) \pi_{\lambda}\left(u_{2}\right)\right)\right| \leq|\lambda|\left\|\pi_{\lambda}(f)\right\|_{2}\left\|\pi_{\lambda}\left(u_{2}\right)\right\|_{2}
$$

is integrable in $\lambda$. Furthermore, note by (3.9) that $\pi_{\lambda}(f)$ has Schwartz kernel $K_{\hat{f}(\cdot, \lambda)}^{\lambda}$ for all $\lambda \neq 0$ and $\pi_{\lambda}(U)$ has kernel $K_{\widehat{U}(\cdot, \lambda)}^{\lambda}$ for a.e. $\lambda$. Thus

$$
\operatorname{tr}\left(\pi_{\lambda}(f) \pi_{\lambda}(U)\right)=\int K_{\hat{f}(\cdot, \lambda)}^{\lambda}(x, y) K_{\widehat{U}(\cdot, \lambda)}^{\lambda}(y, x) d y d x
$$


Hence by (3.10) and (3.11)

$$
\begin{aligned}
\operatorname{tr}\left(\pi_{\lambda}(f) \pi_{\lambda}(U)\right) & =\iint K \hat{f}(x, y, \lambda) K \widehat{U}(y, x, \lambda) d y d x \\
& =\int F_{2} \hat{f}(x-y, \lambda(x+y), \lambda) F_{2} \widehat{U}(y-x, \lambda(y+x), \lambda) d y d x \\
& =\frac{1}{2|\lambda|} \int F_{2} \hat{f}(r, s, \lambda) F_{2} \widehat{U}(-r, s, \lambda) d r d s .
\end{aligned}
$$

Thus by the Plancherel Theorem on $\mathbf{R}$,

$$
\operatorname{tr}\left(\pi_{\lambda}(f) \pi_{\lambda}(U)\right)=\frac{\pi}{|\lambda|} \int \hat{f}(r, s, \lambda) \widehat{U}(-r,-s, \lambda) d r d s
$$

Therefore

$$
\frac{|\lambda|}{2 \pi^{2}} \operatorname{tr}\left(\pi_{\lambda}(f) \pi_{\lambda}(U)\right)=\frac{1}{2 \pi} \iint \hat{f}(r, s, \lambda) \widehat{U}(-r,-s, \lambda) d r d s,
$$

and

$$
\begin{aligned}
\check{U}(f) & =\iiint f(r, s, t) U(-r,-s,-t) d r d s d t \\
& =\frac{1}{2 \pi^{2}} \int|\lambda| \operatorname{tr}\left(\pi_{\lambda}(f) \pi_{\lambda}(U)\right) d \lambda .
\end{aligned}
$$

Now let $P_{n}$ be the orthogonal projection of $L^{2}(\mathbf{R})$ onto the span of the functions $e_{1}, e_{2}, \ldots, e_{n}$. Then by (5.3) and (5.4) and the Lebesgue dominated convergence theorem, $|\lambda| \operatorname{tr}\left(P_{n} \pi_{\lambda}(f) \pi_{\lambda}(U)\right)$ converges in $L^{1}$ to $|\lambda| \operatorname{tr}\left(\pi_{\lambda}(f) \pi_{\lambda}(U)\right)$. But the trace of $P_{n} \pi_{\lambda}(f) \pi_{\lambda}(U)$ equals $\sum_{k=1}^{n} \sum_{m=1}^{n} \widehat{U}_{m, k}(\lambda) \widehat{f}_{k, m}(\lambda)$. The result now follows.

The sums in Theorems 5.1 and 5.2 converge absolutely since they can be reordered arbitrarily. Moreover, the analysis of the operator valued distribution $\widehat{U}$ is now reduced to the study of the ordinary distributions $\widehat{U}_{m, n}$.

The Fourier transform in the case of abelian groups is especially effective in dealing with convolution. This continues to be the case with regard to the distributions $\widehat{U}_{m, n}$. The convolutions of a distribution $U$ and a Schwartz function $f$ are defined by

$$
\begin{aligned}
& (U * f)(z, t)=\int d U(w, s) f\left((w, s)^{-1}(z, t)\right), \\
& (f * U)(z, t)=\int d U(w, s) f\left((z, t)(w, s)^{-1}\right) .
\end{aligned}
$$

We also need the following consequence of Theorem 2.1 .

$$
\int h_{r, s}(z+w, \lambda) \bar{h}_{m, n}(z, \lambda) e^{-i \lambda \sigma(w, z)} d z=\frac{\pi}{|\lambda|} \delta_{r, m} h_{n, s}(w, \lambda) .
$$

Theorem 5.3. Let $f$ be a Schwartz function on $H$ and let $U$ be a tempered distribution. Then

$$
(f * U)_{m, n}^{-}=\sum_{k} \widehat{U}_{m, k} \widehat{f}_{k, n}
$$


and

$$
(U * f)_{m, n}=\sum_{k} \widehat{f}_{m, k} \widehat{U}_{k, n},
$$

where these sums converge weakly.

Proof. By (5.2),

$$
\begin{aligned}
(U & * f)_{m, n}^{-}(\phi)=\iint d(U * f)(z, t) \int \phi(\lambda) h_{m, n}(z, \lambda) e^{-i \lambda t} d \lambda \\
& =\iiint\left(\int d U(w, s) f((-w,-s)(z, t))\right) \phi(\lambda) h_{m, n}(z, \lambda) e^{-i \lambda t} d \lambda d t d z \\
& =\iiint \int d U(w, s) f(z-w, t-s-\sigma(w, z)) e^{-i \lambda t} \phi(\lambda) h_{m, n}(z, \lambda) d t d z d \lambda \\
& =\iiint \int d U(w, s) f(z-w, t) e^{-i \lambda t} d t e^{-i \lambda s} e^{-i \lambda \sigma(w, s)} \phi(\lambda) h_{m, n}(z, \lambda) d z d \lambda \\
& =\iiint d U(w, s) \hat{f}(z-w, \lambda) e^{-i \lambda s} e^{-i \lambda \sigma(w, z)} \phi(\lambda) h_{m, n}(z, \lambda) d z d \lambda .
\end{aligned}
$$

Thus by Theorem 3.2 and by (5.7) and (5.2)

$$
\begin{aligned}
(U * f)_{m, n}(\phi) & =\iiint d U(w, s) \frac{|\lambda|}{\pi} \sum_{p, q} \widehat{f}_{p, q}(\lambda) \bar{h}_{p, q}(z-w, \lambda) h_{m, n}(z, \lambda) \\
& \times \varepsilon^{-i \lambda \sigma(w, z)} d z e^{-i \lambda s} \phi(\lambda) d \lambda \\
& =\iint d U(w, s) \sum_{p, q} \widehat{f}_{p, q}(\lambda) \delta_{m, p} h_{q, n}(w, \lambda) e^{-i \lambda s} \phi(\lambda) d \lambda \\
& =\iint d U(w, s) \sum_{q} \widehat{f}_{m, q}(\lambda) h_{q, n}(w, \lambda) e^{-i \lambda s} \phi(\lambda) d \lambda \\
& =\sum_{q} \iint d U(w, s)\left(\widehat{f}_{m, q}(\lambda) h_{q, n}(w, \lambda) e^{-i \lambda s} \phi(\lambda) d \lambda\right) \\
& =\sum_{q} \int d \widehat{U}_{q, n}(\lambda)\left(\widehat{f}_{m, q}(\lambda) \phi(\lambda)\right)=\sum_{q}\left(\widehat{f}_{m, q} \widehat{U}_{q, n}\right)(\phi) .
\end{aligned}
$$

The other case is handled similarly.

As noted before these results also hold if we use the series of representations $\pi^{\lambda}$ on Fock space. These representations are particularly suited to studying those distributions which have nice covariance properties with respect to rotations on $\mathbf{C}$. This is not surprising for the matrix coefficients

$$
h_{m, n}(z, \lambda)=\left\langle\pi^{\lambda}(z, 0) e_{m}, e_{n}\right\rangle
$$

are covariant under rotations.

For instance, the radial distributions $U$ on $H$ are invariant under rotations. Specifically:

$$
\int d U(z, t) f(u z, t)=\int d U(z, t) f(z, t) \text { if }|u|=1 .
$$


Theorem 5.4. If $U$ is a tempered radial distribution on $H$, then $\widehat{U}_{m, n}=0$ for $m \neq n$.

Proof. By (5.2)

$$
\widehat{U}_{m, n}(\phi)=\int d \widehat{U}(z, \lambda) \phi(\lambda) h_{m, n}(u z, \lambda),
$$

for all $u$. But by $(1.10)$,

$$
h_{m, n}(u z, \lambda)=\left\langle\pi^{\lambda}(u z, 0) e_{m}, e_{n}\right\rangle= \begin{cases}u^{n-m} h_{m, n}(z, \lambda) & \text { if } \lambda>0, \\ \bar{u}^{n-m} h_{m, n}(z, \lambda) & \text { if } \lambda<0 .\end{cases}
$$

Thus $\widehat{U}_{m, n}=0$ if $m \neq n$.

By Theorems 5.3 and 5.5 we have the following corollary.

Corollary 5.6. Let $U$ be a radial tempered distribution on $H$. Then $(f * U)_{m, n}$ $=\widehat{U}_{m, m} \widehat{f}_{m, n}$ and $(U * f)_{m, n}=\widehat{f}_{m, n} \widehat{U}_{n, n}$ for every Schwartz function $f$.

Finally, we remark for a Schwartz function $f$ we have defined a distribution $\widehat{f}_{m, n}$ on $\mathscr{S}_{0}(\mathbf{R})$ and a matrix coefficient $\widehat{f}_{m, n}(\lambda)=\left\langle\pi_{\lambda}(f) e_{m}, e_{n}\right\rangle$ for $\lambda \neq 0$. However, by (5.2) and (3.17) it follows that these are the same. Indeed

$$
\begin{aligned}
\hat{f}_{m, n}(\phi) & =\int \hat{f}(z, \lambda) \int \phi(\lambda) h_{m, n}(z, \lambda) d z \\
& =\int \phi(\lambda)\left(\int \hat{f}(z, \lambda) h_{m, n}(z, \lambda) d z\right) d \lambda \\
& =\int \phi(\lambda) \hat{f}_{m, n}(\lambda) d \lambda .
\end{aligned}
$$

Thus $\hat{f}_{m, n}$ are distributions given by functions. This continues to be the case when $f$ is integrable or square integrable. The rapidity with which these functions decrease at infinity is related to the smoothness of the transforms $\hat{f}_{m, n}$.

\section{CONVOLUTION OPERATORS ON SOBOLEV SPACES}

For each real number $\alpha$ define $\mathscr{H}(\alpha)$ to be the Sobolev space of all complex functions $f$ on $H$ satisfying

$$
\int|f(z, t)|^{2}\left(\left(1+|z|^{2}\right)^{2}+t^{2}\right)^{\alpha} d z d t<\infty
$$

Let $u(z, t)$ be a function on the Heisenberg group $H$. In this section we will study the boundedness of the convolution operator $f \mapsto u * f$ as an operator between the Sobolev spaces $\mathscr{H}(\beta)$ and $\mathscr{H}(-\gamma)$.

Let $x=(z, t) \in H$. Define a norm on $H$ by

$$
|x|=|z|^{4}+t^{2}
$$


Then if $\gamma_{r}(z, t)=\left(r z, r^{2} t\right)$ where $r>0$, then

$$
\left|\gamma_{r} x\right|=r^{4}|x|
$$

and thus $\mid$ is homogeneous of degree 4 with respect to the group of dilations $\gamma_{r}, r>0$. A lemma of Stein and Folland implies there is a constant $C \geq 1$ satisfying

$$
|x y| \leq C(|x|+|y|) .
$$

Define $g_{\alpha}(z, t)=\left(\left(1+|z|^{2}\right)^{2}+t^{2}\right)^{\alpha / 2}$. Then $\mathscr{H}(\alpha)=\left\{f: \int_{H}|f(x)|^{2} g_{2 \alpha}(x) d x\right.$ $<\infty\}$. Moreover, if

$$
J_{\alpha} f=g_{\alpha} f,
$$

then $J_{\alpha}$ is an isometry of $\mathscr{H}(\beta)$ onto $\mathscr{H}(\beta-\alpha)$. From (6.3) one has

$$
g_{\alpha}(x y) \leq(2+C)^{\alpha / 2} g_{\alpha}(x) g_{\alpha}(y) .
$$

Furthermore, the natural Hermitian pairing between $\mathscr{H}(-\alpha)$ and $\mathscr{H}(\alpha)$ defined by $\langle f, h\rangle_{\alpha}=\int f(x) \overline{h(x)} d x$ satisfies

$$
\left|\langle f, h\rangle_{\alpha}\right| \leq\|f\|_{-\alpha}\|h\|_{\alpha} .
$$

Lemma 6.1. Suppose $\beta>1, \gamma>1, \alpha<\beta-1$, and $\alpha<\gamma-1$. Then

$$
\iint g_{\alpha}^{2}(x y) g_{-\beta}^{2}(x) g_{-\gamma}^{2}(y) d x d y<\infty
$$

Proof. We do two cases.

Case 1: $\alpha \geq 0$. By 6.5 one has

$$
\begin{aligned}
& \iint_{H \times H} g_{\alpha}^{2}(x y) g_{-\beta}^{2}(x) g_{-\gamma}^{2}(y) d x d y \\
& \quad \leq(2+C)^{\alpha} \int g_{2 \alpha-2 \beta}(x) d x \int g_{2 \alpha-2 \gamma}(y) d y<\infty
\end{aligned}
$$

if $\alpha<\beta-1$ and $\alpha<\gamma-1$.

Case 2: $\alpha \leq 0$. Then,

$$
\iint g_{\alpha}^{2}(x y) g_{-\beta}^{2}(x) g_{-\gamma}^{2}(y) d x d y<\infty
$$

if

$$
\int g_{-\beta}^{2}(x) d x<\infty \text { and } \int g_{-\gamma}^{2}(y) d y<\infty .
$$

This occurs if $\beta>1$ and $\gamma>1$.

Proposition 6.2. Let $\beta>1, \gamma>1, \alpha<\beta-1$, and $\alpha<\gamma-1$. Then $f \mapsto g_{\alpha} * f$ is a bounded operator from $\mathscr{H}(\beta)$ into $\mathscr{H}(-\gamma)$.

Proof. Define $L_{x} g(y)=g\left(x^{-1} y\right)$. Then $g_{\alpha} * f(x)=\left\langle\check{f}, L_{x} g_{\alpha}\right\rangle_{\beta}$ where $\check{f}(y)=$ $f\left(y^{-1}\right)$. Thus by (6.6), $\left|f * g_{\alpha}(x)\right| \leq\|f\|_{\beta}\left\|L_{x} g_{\alpha}\right\|_{-\beta}$. 
Set $H(x)=\left\|L_{x} g_{\alpha}\right\|_{-\beta}$. Then by Lemma 6.1

$$
\begin{aligned}
\|H\|_{-\gamma} & =\int H(x)^{2} g_{-\gamma}^{2}(x) d x \\
& =\iint g_{\alpha}^{2}\left(x^{-1} y\right) g_{-\beta}^{2}(y) g_{-\gamma}^{2}(x) d y d x \\
& =\iint g_{\alpha}^{2}(x y) g_{-\beta}^{2}(y) g_{-\gamma}^{2}(x) d y d x<\infty .
\end{aligned}
$$

Note that $\mathscr{H}(\beta) \supseteq \mathscr{H}(\alpha)$ for $\beta<\alpha$. Hence we define the left limit Sobolev space at $\alpha$ by

$$
\mathscr{H}(\alpha-)=\bigcap_{\beta<\alpha} \mathscr{H}(\beta)
$$

Theorem 6.3. Let $\beta>\frac{1}{2}, \gamma>\frac{1}{2}$ and $\alpha<\beta-\frac{1}{2}, \alpha<\gamma-\frac{1}{2}$. Then if $u \in$ $\mathscr{H}(-\alpha-)$, then the mapping $f \mapsto u * f$ is a bounded operator from $\mathscr{H}(\beta)$ into $\mathscr{H}(\gamma)$.

Proof. Note $|u * f(x)| \leq \int|u(x y)|\left|f\left(y^{-1}\right)\right| d y$. Hence we may assume both the functions $u$ and $f$ are nonnegative. Moreover, by the Cauchy-Schwarz inequality,

$$
\begin{aligned}
|u * f(x)|^{2} & =\left(\int g_{r}(x y)\left(J_{-\beta} f\right)^{1 / 2}\left(y^{-1}\right) g_{-r}(x y) u(x y)\left(J_{\beta} f\right)^{1 / 2}\left(y^{-1}\right) d y\right)^{2} \\
& \leq\left(\int g_{2 r}(x y) J_{-\beta} f\left(y^{-1}\right) d y\right)\left(g_{-2 r}(x y) u(x y)^{2} J_{\beta} f\left(y^{-1}\right) d y\right) .
\end{aligned}
$$

Thus $|u * f(x)|^{2} \leq A(x) B(x)$ where

$$
A(x)=\left(g_{2 r} * J_{-\beta} f\right)(x) \text { and } B(x)=\left(J_{-r} u\right)^{2} * J_{\beta} f(x) .
$$

Choose $r>\alpha$ satisfying $2 r<2 \beta-1$ and $2 r<2 \gamma-1$. By (6.4) and Proposition 6.2, there is a constant $K>0$ with

$$
\|A\|_{-2 \gamma} \leq K\left\|J_{-\beta} f\right\|_{2 \beta}=K\|f\|_{\beta} .
$$

Moreover, by (6.4), $J_{\beta} f \in \mathscr{H}(0)$ and $J_{-r} u \in \mathscr{H}(r-\alpha-)$ for $u \in \mathscr{H}(-\alpha-)$. Therefore $\left(J_{-r} u\right)^{2} \in L^{1}$ and

$$
\|B\|_{0} \leq\left\|J_{-r} u\right\|_{0}^{2}\left\|J_{\beta} f\right\|_{0} \leq\left\|J_{-r} u\right\|_{0}^{2}\|f\|_{\beta} .
$$

Thus by (6.4), (6.6), (6.8), (6.9), and (6.10):

$$
\begin{aligned}
\|u * f\|_{-\gamma}^{2} & =\int|u * f(x)|^{2} g_{-2 \gamma}(x) d x \\
& \leq \int A(x) J_{-2 \gamma} B(x) d x=\left\langle A, J_{-2 \gamma} B(x)\right\rangle_{2 \gamma} \\
& \leq\|A\|_{-2 \gamma}\left\|J_{-2 \gamma} B\right\|_{2 \gamma} \leq K\left\|J_{-r} u\right\|_{0}^{2}\|f\|_{\beta}^{2} .
\end{aligned}
$$




\section{REPRESENTATIONS OF $\mathrm{SU}(2,1)$}

In this part of the paper we develop techniques which allow one to determine unitary structures on some subquotients of the nonunitary principal series. Essentially we show at a reductive point of the principal series where the intertwining operator has a null space, there is a chain of null spaces corresponding to a sequence of intertwining operators with decreasing domains. On each quotient space of the successive null spaces, there is an invariant form which if strictly definite on the quotient determines a unitary structure for the quotient. For the group $\mathrm{SO}(4,1)$, it was shown in [5] that the sequence can only have length at most one, and that this process along with the unitary principal series and complementary series yield all the irreducible unitary representations of $\mathrm{SO}(4,1)$. For the group $\mathrm{SU}(2,1)$, the sequence may have length one or two. Moreover, using results of Kraljevic [9] it can be shown again one obtains most of the nonprincipal unitary and complimentary series dual. It is our intent to investigate in a later paper precisely which can be obtained.

The approach taken in this part of the paper is to analytically describe the convolution operators on the Sobolev spaces $\mathscr{H}(\alpha)$ that define the intertwining operators and use the techniques on distributions obtained in Part I to yield trace integral descriptions of the invariant forms on the null-space representations mentioned above. This description is quite useful. It allows one to determine for the unitary subquotients the spectral behavior of these representations on the minimal parabolic subgroup $M A \bar{N}$. For example, most have infinite multiplicity on $\bar{N}$, but there are examples of uniform multiplicity one and a mixture of one and infinite multiplicity on $\bar{N}$. Moreover there are examples of subquotients that are irreducible on $M A \bar{N}$. This never occurs for a unitary principal series or complementary series representation.

\section{Decompositions of $\mathrm{SU}(2,1)$}

Let $G=\mathrm{SU}(2,1)$. In this section we establish the setup in which we will analyze the principal series of $G$.

The Cartan involution $\theta$ on $G$ is defined by $\theta(g)=\left(g^{-1}\right)^{*}$. Let $K$ be the fixed point set of $\theta$. Then $K$ is a maximal compact subgroup of $G$. If $A$ is the subgroup $\exp (\mathbf{R} h)$ where

$$
h=\left[\begin{array}{lll}
0 & 0 & 0 \\
0 & 0 & 1 \\
0 & 1 & 0
\end{array}\right],
$$

then $A$ is a maximal abelian subgroup of the space $P=\left\{g: \theta(g)=g^{-1}\right\}$. The centralizer $M$ of $A$ in $K$ consists of all matrices

$$
u=\left[\begin{array}{ccc}
u^{2} & 0 & 0 \\
0 & \bar{u} & 0 \\
0 & 0 & \bar{u}
\end{array}\right],
$$

where $|u|=1$. 
Define $\bar{N}$ to be the group of matrices having form

$$
(z, t)=\left[\begin{array}{ccc}
1 & z & z \\
-\bar{z} & 1-\frac{1}{2}|z|^{2}+t i & -\frac{1}{2}|z|^{2}+t i \\
-\bar{z} & \frac{1}{2}|z|^{2}+t i & 1+\frac{1}{2}|z|^{2}-t i
\end{array}\right] .
$$

This group is identified with the Heisenberg group defined in (I.1.1).

Set $N=\theta(\bar{N})$; then $N$ is also isomorphic to the Heisenberg group $H$. The Iwasawa decomposition is

$$
G=N A K
$$

Moreover, using the Bruhat decomposition, one has $N A M \bar{N}$ is an open submanifold of $G$ whose complement is the set $N A M w^{*}$ where

$$
w^{*}=\left[\begin{array}{ccc}
-1 & 0 & 0 \\
0 & -1 & 0 \\
0 & 0 & 1
\end{array}\right]
$$

is a representative of the nontrivial element in the Weyl group $M^{*} / M$, where $M^{*}$ is the normalizer of the group $A$ in $K$.

Define $a(r)=\exp (\log r h)$ for $r>0$. Then:

$$
\begin{gathered}
a(r)(z, t) a(r)^{-1}=\left(r^{-1} z, r^{-2} t\right), \\
a(r) \theta(z, t) a(r)^{-1}=\left(r z, r^{2} t\right),
\end{gathered}
$$

and

$$
\begin{gathered}
u(z, t) u^{-1}=\left(u^{3} z, t\right), \\
u \theta(z, t) u^{-1}=\theta\left(u^{3} z, t\right) .
\end{gathered}
$$

Let $\mathscr{L}_{N}$ be the Lie algebra of $N$. For $x \in \mathscr{L}_{A}$, the Lie algebra of $A$, set $\rho(x)=\frac{1}{2} \operatorname{tr}\left(\left.\operatorname{ad} x\right|_{\mathscr{L}_{N}}\right)$. By (1.6), one has

$$
\rho(h)=2 \text {. }
$$

\section{THE PRINCIPAL SERIES}

Let $\alpha$ be a complex valued linear functional on $\mathscr{L}_{A}$ and let $\chi$ be a character on $M$. Then we identify $\alpha$ with the complex number $\alpha(h)$ and $\chi$ with the integer $p$ for which $\chi(u)=u^{p}$.

Set $\mathscr{H}^{p, \alpha}$ to be the Hilbert space of measurable complex valued functions $f$ on $G$ satisfying

$$
f(\text { namg })=e^{(\alpha+p)(\log a)} \chi(m) f(g)
$$

and

$$
\int|f(k)|^{2} d k<\infty
$$


The principal series representation $R^{p, \alpha}$ is defined by

$$
R^{p, \alpha}(g) f(x)=f(x g) .
$$

These representations are strongly continuous and are unitary when $\operatorname{Re} \alpha=0$. By Proposition 6.4 of [8] the representation $R^{p, \alpha}$ at the Casimir element $C$ satisfies

$$
R^{p, \alpha}(C)=\frac{1}{36}\left(p^{2}+3 \alpha^{2}-12\right)
$$

It follows that $R^{p, \alpha}$ can have unitary subquotients only if $\alpha$ is either real or pure imaginary. But if $\alpha$ is pure imaginary, the representation $R^{p, \alpha}$ is in the principal unitary series. Thus we need only consider the case when $\alpha$ is real. By Theorem 7 of [9] those irreducible unitary representations not occurring within the unitary principal series occur as subquotients of $R^{p, \alpha}$ for some $\alpha>0$.

Now $N A M \bar{N}$ is a conull open subset of $G$. One then may consider the Hilbert space $\mathscr{H}^{p, \alpha}$ as functions on $\bar{N}$. Using standard arguments, e.g. [18], involving explicit formulas for the $N A M \bar{N}$ decomposition of $G,\left.f \mapsto f\right|_{\bar{N}}$ defines an isometry of $\mathscr{H}^{p, \alpha}$ onto $\mathscr{H}(\alpha)=L^{2}\left(H,\left(\left(1+|z|^{2}\right)^{2} / 2+t^{2}\right)^{\alpha} d z d t\right)$, where here Haar measure on $K$ is chosen so that no constants are necessary in the isometry.

The representation $R^{p, \alpha}$ on $\mathscr{H}(\alpha)$ becomes

$$
R^{p, \alpha}(g) f(h)=e^{(\alpha+\rho)(\log a)} \chi(u) f\left(h^{\prime}\right),
$$

if $h g=n a u h^{\prime}$.

On $M A \bar{N}$ one has

$$
\begin{gathered}
R^{p, \alpha}\left(h_{1}\right) f\left(h_{2}\right)=f\left(h_{2} h_{1}\right), \\
R^{p, \alpha}(a(r)) f(z, t)=r^{\alpha+2} f\left(r z, r^{2} t\right),
\end{gathered}
$$

and

$$
R^{p, \alpha}(u) f(z, t)=u^{p} f\left(u^{-3} z, t\right)
$$

The natural Hermitian pairing (I.6.6) between $\mathscr{H}(-\alpha)$ and $\mathscr{H}(\alpha)$ gives an invariant pairing between $R^{p,-\alpha}$ and $R^{p, \alpha}$. Namely,

$$
\left\langle R^{p,-\alpha}(g) f_{1}, R^{p, \alpha}(g) f_{2}\right\rangle_{\alpha}=\left\langle f_{1}, f_{2}\right\rangle_{\alpha} \text { if } f_{1} \in \mathscr{H}(-\alpha) \text { and } f_{2} \in \mathscr{H}(\alpha) .
$$

\section{INTERTWINING OPERATORS}

The intertwining operators are defined formally on $\mathscr{H}^{p, \alpha}$ by

$$
A(p, \alpha) f(z, t)=\int f\left(w^{*} \theta(w, s)^{-1}(z, t)\right) d w d s .
$$

By results from [17], it is known that these integrals define bounded operators when $\operatorname{Re} \alpha>0$ and

$$
R^{p,-\alpha}(g) A(p, \alpha)=A(p, \alpha) R^{p, \alpha}(g) .
$$


Hence, they are bounded when considered as operators between $\mathscr{H}(\alpha)$ and $\mathscr{H}(-\alpha)$. Furthermore, using (2.1) to extend the integrand in (3.1) one sees

$$
\begin{aligned}
A(p, \alpha) f(z, t)=\iint\left(\frac{1}{4}|w|^{4}+s^{2}\right)^{\alpha / 2-1} & {\left[\frac{\frac{1}{2}|w|^{2}-s i}{\left.\left|\frac{1}{2}\right| w\right|^{2}-s i \mid}\right]^{p} } \\
& \times f\left((w, s)^{-1}(z, t)\right) d w d s .
\end{aligned}
$$

Thus $A(p, \alpha) f=U * f$ where $U$ is the distribution on $H$ defined by the function

$$
U(z, t)=\left(\frac{1}{4}|z|^{4}+t^{2}\right)^{\alpha / 2-1}\left[\frac{\frac{1}{2}|z|^{2}-t i}{\left.\left|\frac{1}{2}\right| z\right|^{2}-t i \mid}\right]^{p} .
$$

These distributions are homogeneous with respect to the group of dilations $\gamma_{r}$ defined in $\S 6$ of Part I. Namely

$$
d U\left(\gamma_{r}(z, t)\right)=r^{2 \alpha} d U(z, t) .
$$

Moreover, note $U$ depends analytically on $\alpha$ and $U$ is locally $L^{1}$ at $(0,0)$ for $\alpha>0$. Using this one can see that

$$
U=u_{1}+u_{2} \text { where } u_{1} \in L^{2}(H) \text { and } u_{2} \in L^{2}(H) \text { if } 0<\alpha<1,
$$

and

$$
U \in \mathscr{H}(-(\alpha-1)-) \text { if } \alpha>1
$$

Thus by (I.6.3)

$$
\begin{aligned}
& A(p, \alpha) \text { is a bounded convolution operator from } \mathscr{H}(\beta) \text { into } \\
& \mathscr{H}(\gamma) \text { if } \alpha>1, \beta>\alpha-\frac{1}{2} \text {, and } \gamma>\alpha-\frac{1}{2} .
\end{aligned}
$$

Since $U(u w, s)=U(w, s)$ when $|u|=1, U$ defines a radial tempered distribution on $H$. By Theorems I.5.3 and I.5.4, one has

$$
\widehat{U}_{m, n}=0 \text { if } m \neq n
$$

and

$$
(U * f)_{m, n}=\widehat{f}_{m, n} \widehat{U}_{n, n} .
$$

Note by (3.6) that $U$ is the sum of an $L^{1}$ and an $L^{2}$ function if $0<\alpha<1$. Hence $\pi^{\lambda}(U)$ is defined a.e. $\lambda$.

Proposition 3.1. Suppose $0<\alpha<1$ and $\phi \in \mathscr{S}_{0}(H)$. Then

$$
\widehat{U}(\phi)=\int \phi(\lambda) A(\lambda) d \lambda
$$

where

$$
A(\lambda)=\pi^{\lambda}(U)=\iint U(z, t) \pi^{\lambda}(z, t) d z d t
$$


Moreover,

Proof. By (I.4.1)

$$
\|A(\lambda)\| \leq\left\|u_{1}\right\|_{1}+\left(\frac{\pi}{|\lambda|} \int\left|\widehat{u}_{2}(z, \lambda)\right|^{2} d z\right)^{1 / 2} .
$$

$$
\widehat{U}(\phi)=\iiint \phi(\lambda) U(z, t) \pi^{\lambda}(z, t) d \lambda d z d t .
$$

Note

$$
\left\|\int u_{1}(z, t) \pi^{\lambda}(z, t) d z d t\right\| \leq\left\|u_{1}\right\|_{1},
$$

and by (I.2.5) and (I.3.3) that

$$
\iint u_{2}(z, t) \pi^{\lambda}(z, t) d z d t=\rho^{\lambda}\left(\widehat{u}_{2}(\cdot, \lambda)\right)
$$

But by (I.3.5)

$$
\left\|\rho^{\lambda}\left(\widehat{u}_{2}(\cdot, \lambda)\right)\right\|^{2} \leq \frac{\pi}{|\lambda|} \int\left|\widehat{u}_{2}(z, \lambda)\right|^{2} d z
$$

Thus

$$
\widehat{U}(\phi)=\int \phi(\lambda)\left(\iint u(z, t) \pi^{\lambda}(z, t) d z d t\right) d \lambda=\int \phi(\lambda) A(\lambda) d \lambda .
$$

Note $A(\lambda)$ is a bounded operator on $\mathscr{H}$ if $\lambda<0$ and is bounded on $\overline{\mathscr{H}}$ when $\lambda>0$. Define

$$
\widehat{u}_{m, m}(\lambda)= \begin{cases}\left\langle A(\lambda) e_{m}, e_{m}\right\rangle & \text { if } \lambda>0 \\ \left\langle A(\lambda) e_{m}, e_{m}\right\rangle^{-} & \text {if } \lambda<0\end{cases}
$$

Since $\widehat{U}_{m, m}(\phi)=\left\langle\widehat{U}(\phi) e_{m}, e_{m}\right\rangle$, it follows from Proposition 3.1 that

$$
\widehat{U}_{m, m}(\phi)=\int \phi(\lambda) \widehat{u}_{m, m}(\lambda) d \lambda .
$$

Next we diagonalize the operators $A(\lambda)$ relative to the basis $e_{m}$. Such a diagonalization is possible since $\widehat{U}_{m, n}=0$ for $m \neq n$ is equivalent to the diagonalizability of $A(\lambda)$ relative to the basis $\left\{e_{m}\right\}$. Moreover, $\widehat{u}_{m, m}(\lambda)$ give the diagonal entries.

Note by (I.5.5) and the remarks after Theorem I.5.2 that for $0<\alpha<1$ one has

$$
\check{U}(f)=\frac{1}{2 \pi^{2}} \int|\lambda| \operatorname{tr}\left(\pi^{\lambda}(f) A(\lambda)\right) d \lambda
$$

for all Schwartz functions $f$.

Furthermore, (I.1.8) shows

$$
\pi^{\lambda}=\pi \circ \gamma_{\sqrt{\lambda}} \text { and } \pi^{-\lambda}=\bar{\pi} \circ \gamma_{\sqrt{\lambda}} \text { for } \lambda>0 .
$$


Therefore, if $\lambda>0$,

$$
\begin{aligned}
A(\lambda) & =\int U(z, t) \pi \circ \gamma_{\sqrt{\lambda}}(z, t) d z d t \\
& =\int d U(z, t) \pi \circ \gamma_{\sqrt{\lambda}}(z, t)=\int d U\left(\gamma_{\sqrt{\lambda^{-1}}}(z, t)\right) \pi(z, t) .
\end{aligned}
$$

Thus by (3.6) we see

$$
A(\lambda)= \begin{cases}\pi(U) / \lambda^{\alpha} & \text { if } \lambda>0 \\ \bar{\pi}(U) /|\lambda|^{\alpha} & \text { if } \lambda<0\end{cases}
$$

Hence by (3.13) and (3.15) we have:

Proposition 3.2. Suppose $0<\alpha<1$. Then:

(a) $\check{U}(f)=\frac{1}{2 \pi^{2}}\left(\int_{-\infty}^{0} \operatorname{tr}\left(\pi^{\lambda}(f) \bar{\pi}(U)\right)|\lambda|^{1-\alpha} d \lambda\right.$

$$
\left.+\int_{0}^{\infty} \operatorname{tr}\left(\pi^{\lambda}(f) \pi(U)\right)|\lambda|^{1-\alpha} d \lambda\right)
$$

and

(b)

$$
\widehat{u}_{n, n}(\lambda)= \begin{cases}|\lambda|^{-\alpha}\left\langle\pi(U) e_{n}, e_{n}\right\rangle & \text { for } \lambda>0, \\ |\lambda|^{-\alpha}\left\langle\bar{\pi}(U) e_{n}, e_{n}\right\rangle & \text { for } \lambda<0\end{cases}
$$

To calculate the matrix coefficient $\left\langle\pi(U) e_{n}, e_{n}\right\rangle$, note by (I.1.6) that:

$$
\begin{aligned}
\left\langle\pi(U) e_{n}, e_{n}\right\rangle & =\iint U(z, t) e^{-i t}\left\langle\pi(z, 0) e_{n}, e_{n}\right\rangle d z d t \\
& =\iint U(z, t) e^{-i t} L_{n}^{0}\left(|z|^{2}\right) e^{-|z|^{2} / 2} d z d t .
\end{aligned}
$$

Thus by (3.4) and a change to polar coordinates, one has

$$
\begin{aligned}
\left\langle\pi(U) e_{n}, e_{n}\right\rangle=2 \pi \iint_{0}^{\infty}\left(\frac{1}{4} r^{4}+t^{2}\right)^{\alpha / 2-1} & \left(\frac{\frac{1}{2} r^{2}-i t}{\left|\frac{1}{2} r^{2}-i t\right|}\right)^{p} \\
& \times e^{-i t} L_{n}^{0}\left(r^{2}\right) e^{-r^{2} / 2} r d r d t .
\end{aligned}
$$

The change of variables $r=\sqrt{2 x}, t=x y$ yields

$$
\begin{aligned}
&\left\langle\pi(U) e_{n}, e_{n}\right\rangle=2 \pi \int_{-\infty}^{\infty}\left(\int_{0}^{\infty} x^{\alpha-1} e^{-x(1+i y)} L_{n}^{0}(2 x) d x\right) \\
& \times\left(1+y^{2}\right)^{\alpha / 2-1}\left(\frac{1-i y}{|1-i y|}\right)^{p} d y
\end{aligned}
$$

But by (6.1.1) of [1]

$$
\int_{0}^{\infty} t^{z-1} e^{-k t} d t=k^{-z} \Gamma(z) \text { if } \operatorname{Re} z>0 \text { and } \operatorname{Re} k>0
$$


Thus

$$
\begin{aligned}
\int_{0}^{\infty} x^{\alpha-1} e^{-x(1+i y)} L_{n}^{0}(2 x) d x & =\sum_{l=0}^{n}(-1)^{l} \frac{2^{l} n !}{(n-l) !(l !)^{2}} \int_{0}^{\infty} x^{\alpha+l-1} e^{-x(1+i y)} d x \\
& =\sum_{l=0}^{n}(-1)^{l} \frac{2^{l} n !}{(n-l) !(l !)^{2}}(1+i y)^{\alpha+l} \Gamma(\alpha+l) .
\end{aligned}
$$

It follows by (3.18) that

$$
\begin{aligned}
\left\langle\pi(U) e_{n}, e_{n}\right\rangle=2 \pi n ! & \sum_{l=0}^{n} \frac{(-2)^{l} \Gamma(\alpha+l)}{(n-l) !(l !)^{2}} \\
& \times \int_{-\infty}^{\infty}(1+i y)^{-\alpha / 2-p / 2-l-1}(1-i y)^{\alpha / 2+p / 2-1} d y
\end{aligned}
$$

Define $I(r, s)$ by

$$
I(r, s)=\int_{-\infty}^{\infty}(1+i t)^{-r}(1-i t)^{-s} d t \text { when } r+s>1 .
$$

Then by (3.20) one has

$$
\left\langle\pi(U) e_{n}, e_{n}\right\rangle=2 \pi n ! \sum_{l=0}^{n} \frac{(-2)^{l} \Gamma(\alpha+l)}{(n-l) !(l !)^{2}} I\left(\frac{\alpha}{2}+\frac{p}{2}+l+1,1-\frac{\alpha}{2}-\frac{p}{2}\right) .
$$

Proposition 3.3. Suppose $r+s>1$. Then

$$
I(r, s)=\frac{4 \pi}{2^{r+s}(r+s-1) B(r, s)}
$$

where

$$
B(r, s)=\frac{\Gamma(r) \Gamma(s)}{\Gamma(r+s)}
$$

is the Beta function.

Proof. Define $f_{l}$ by

$$
f_{l}(\lambda)= \begin{cases}\lambda^{l} e^{-\lambda} & \text { if } \lambda>0 \\ 0 & \text { if } \lambda<0\end{cases}
$$

Then for $\lambda>0, f_{l} \in L^{1}(\mathbf{R})$ and by (3.19), $\widehat{f}_{l}(t)=(1+i t)^{-l-1} \Gamma(l+1)$.

Moreover, by the Plancherel Theorem on $\mathbf{R}$ one has

$$
\frac{1}{2 \pi} \int \hat{f}(t) \hat{g}(-t) d t=\int f(\lambda) g(\lambda) d \lambda \text {. }
$$

Therefore

$$
\begin{aligned}
\int_{0}^{\infty} \lambda^{r-1} e^{-\lambda} \lambda^{s-1} e^{-\lambda} d \lambda & =\frac{1}{2 \pi} \int \Gamma(r) \Gamma(s)(1+i t)^{-r}(1-i t)^{-s} d t \\
& =\frac{1}{2 \pi} \Gamma(r) \Gamma(s) I(r, s) \quad \text { if } r>1 \text { and } s>1
\end{aligned}
$$


Thus by (3.19)

$$
\begin{aligned}
I(r, s) & =\frac{2 \pi}{\Gamma(r) \Gamma(s)} \int_{0}^{\infty} \lambda^{r+s-1} e^{-2 \lambda} d \lambda \\
& =\frac{2 \pi}{\Gamma(r) \Gamma(s)} 2^{r+s-1} \Gamma(r+s-1)=2^{r+s} \pi \frac{\Gamma(r+s)}{(r+s-1) \Gamma(r) \Gamma(s)},
\end{aligned}
$$

for $r>1$ and $s>1$. The result follows by analytic continuation.

To continue the calculation we shall need the following identity of Vandermonde:

$$
\frac{\Gamma(\alpha) \Gamma(w+n-2)}{\Gamma(w-\alpha) \Gamma(w+n) n !}=\sum_{l=0}^{n}(-1)^{l} \frac{\Gamma(\alpha+l)}{\Gamma(w+l) l !(n-l) !} .
$$

By (3.22), Proposition 3.3, and (3.23) one has

$$
\begin{aligned}
\left\langle\pi(U) e_{n}, e_{n}\right\rangle & =2 \pi^{2} n ! \sum_{l=0}^{n} \frac{(-1)^{l} \Gamma(\alpha+l)}{(n-l) ! l ! \Gamma(\alpha / 2+p / 2+l+1) \Gamma(1-\alpha / 2-p / 2)} \\
& =\frac{2 \pi^{2} \Gamma(\alpha) \Gamma(1+n+p / 2-\alpha / 2)}{\Gamma(p / 2-\alpha / 2+1) \Gamma(1-\alpha / 2-p / 2) \Gamma(\alpha / 2+p / 2+n+1)}
\end{aligned}
$$

Next notice

$$
\begin{aligned}
\left\langle\bar{\pi}(U) e_{n}, e_{n}\right\rangle^{-} & =\iint U(z, t) \overline{\left\langle\pi(z, t) e_{n}, e_{n}\right\rangle} d z d t \\
& =\iint U(z, t) e^{i t \overline{\left\langle\pi(z, 0) e_{n}, e_{n}\right\rangle}} d z d t \\
& =\iint U(z,-t) e^{-i t \overline{\left\langle\pi(z, 0) e_{n}, e_{n}\right\rangle}} d z d t \\
& =\left\langle\pi(\bar{U}) e_{n}, e_{n}\right\rangle .
\end{aligned}
$$

Since $\bar{U}$ has the same parameters as $U$ except that $p$ is replaced by $-p$, one has

$$
\left\langle\pi(\bar{U}) e_{n}, e_{n}\right\rangle=\frac{2 \pi^{2} \Gamma(\alpha) \Gamma(1+n-p / 2-\alpha / 2)}{\Gamma(p / 2-\alpha / 2+1) \Gamma(1-\alpha / 2-p / 2) \Gamma(\alpha / 2-p / 2+n+1)} .
$$

Define $c_{n}^{ \pm}(\alpha)$ by

$$
c_{n}^{ \pm}(\alpha)=\frac{2 \pi^{2} \Gamma(\alpha) \Gamma(1+n \pm p / 2-\alpha / 2)}{\Gamma(p / 2-\alpha / 2+1) \Gamma(1-\alpha / 2-p / 2) \Gamma(\alpha / 2 \pm p / 2+n+1)} .
$$

From Proposition 3.2 we then have for $0<\alpha<1$

$$
u_{n, n}(\lambda)= \begin{cases}c_{n}^{+}(\alpha) / \lambda^{\alpha} & \text { if } \lambda>0 \\ c_{n}^{-}(\alpha) /|\lambda|^{\alpha} & \text { if } \lambda<0\end{cases}
$$

Moreover, the operators $A(\lambda)$ satisfy $A(\lambda) e_{n}=\widehat{u}_{n, n}(\lambda) e_{n}$ for these $\alpha$. 
Formulas (3.14) and (3.25) completely determine the distributions $\widehat{U}_{n, n}$ when the parameter $\alpha$ is in the interval $(0,1)$. Furthermore, the distribution $\check{U}$ may be calculated using the integral trace formula in Proposition 3.2 for $\alpha$ in this interval.

The functions $c_{n}^{ \pm}$are defined for all $\alpha>0$. We hence define

$$
\widehat{u}_{n, n}(\lambda)= \begin{cases}c_{n}^{+}(\alpha) /|\lambda|^{\alpha} & \text { if } \lambda>0, \\ c_{n}^{-}(\alpha) / \lambda^{\alpha} & \text { if } \lambda<0 .\end{cases}
$$

Define $A(\lambda)$ by

$$
A(\lambda) e_{n}=\widehat{u}_{n, n}(\lambda) e_{n},
$$

where $A(\lambda)$ is a linear operator on $\mathscr{H}$ when $\lambda$ is positive and where it is linear of $\overline{\mathscr{H}}$ when $\lambda$ is negative. As seen from Proposition 3.1, we have $A(\lambda)=\pi^{\lambda}(U)$ when $\alpha$ is between 0 and 1 . By analyticity of the matrix coefficients of $A(\lambda)$ with respect to $\alpha$ we can consider $A(\lambda)$ as an analytic extension of the operators $\pi^{\lambda}(U)$. The operators $\pi^{\lambda}(U)$ are not bounded for $\alpha \geq 1$ but as can be seen from (3.24), (3.26), and (3.27), the operators $A(\lambda)$ are bounded for all $\alpha>0$. These operators are the principal bounded parts of the distributions $\pi^{\lambda}(U)$. We shall also use the notation $\pi_{b}^{\lambda}(U)$ to denote $A(\lambda)$ and $\pi_{b}=\pi_{b}^{1}$. We thus have $\pi^{\lambda}(U)=\pi_{b}^{\lambda}(U)$ provided $0<\alpha<1$. Indeed we have:

Proposition 3.4. Suppose $\hat{f} \in \mathscr{S}_{0}(H)$ and $\phi \in \mathscr{S}_{0}(\mathbf{R})$. Then for $\alpha>0$

(a) $\quad \check{U}(f)=\frac{1}{2 \pi^{2}}\left(\int_{-\infty}^{0} \operatorname{tr}\left(\pi^{\lambda}(f) \bar{\pi}_{b}(U)\right)|\lambda|^{1-\alpha} d \lambda\right.$

$$
\left.+\int_{0}^{\infty} \operatorname{tr}\left(\pi^{\lambda}(f) \pi_{b}(U)\right)|\lambda|^{1-\alpha} d \lambda\right)
$$

(b)

$$
\widehat{U}_{n, n}(\phi)=\int \phi(\lambda) \widehat{u}_{n, n}(\lambda) d \lambda
$$

Proof. $\check{U}(f)$ is an analytic function of $\alpha$ for $\alpha>0$. By Proposition 3.2 (a) holds for $0<\alpha<1$. But the right-hand side of the equality in (a) is also analytic in $\alpha$ for $\alpha>0$. Hence the equality holds for all $\alpha>0$. The second statement is proved similarly.

We remark that from Theorem I.5.4 one has

$$
(A(p, \alpha) f)_{m, n}=\widehat{f}_{m, n} U_{n, m},
$$

if $f$ is a Schwartz function on $H$. By continuity of the operator $A(p, \alpha)$ and the $L^{2}$ density of the Schwartz functions, this identity holds for all $f$ in $\mathscr{H}(\alpha)$.

\section{REPRESENTATIONS OF $M A \bar{N}$}

In this section we describe the unitary dual of the minimal parabolic subgroup $P=M A \bar{N}$. We also establish equivalences of each infinite-dimensional unitary 
representation of $P$ which is nontrivial on the center of $\bar{N}$ with all members of a family of representations of $P$ defined in terms of the matrix coefficients of $\pi$ on $H$ and a real parameter $\alpha$. These equivalences will be used to obtain the spectral decompositions of restrictions to $P$ of representations of $\mathrm{SU}(2,1)$.

Define a unitary operator $T_{u},|u|=1$, on $\mathscr{H}$ and $\overline{\mathscr{H}}$ by

$$
T_{u} f(z)=f\left(u^{-3} z\right) \text {. }
$$

An easy calculation using (I.1.3) shows

$$
T_{u} \pi(z, t) T_{u}^{-1}=\pi\left(u^{3} z, t\right)
$$

and similarly

$$
T_{u} \bar{\pi}(z, t) T_{u}^{-1}=\bar{\pi}\left(u^{3} z, t\right) .
$$

Suppose $p$ is an integer. Define $\tau^{p,+}$ and $\tau^{p,-}$ on $L^{2}((0, \infty), \mathscr{H})$ and $L^{2}((-\infty, 0), \overline{\mathscr{H}})$ respectively by

$$
\begin{aligned}
\tau^{p, \pm}(h) f(\lambda) & =\pi^{\lambda}(h) f(\lambda), \\
\tau^{p, \pm}(a(r)) f(\lambda) & =r^{-1} f\left(r^{-2} \lambda\right), \\
\tau^{p, \pm}(u) f(\lambda) & =u^{p} T_{u} f(\lambda) .
\end{aligned}
$$

By (1.5), (1.7), (I.1.3), (I.1.8), (4.2), and (4.3) the $\tau^{p, \pm}$ are unitary representations of $P$. Moreover, by (4.4) one has

$$
\left.\tau^{p,+}\right|_{H}=\int_{0}^{\infty} \pi^{\lambda} d \lambda
$$

and

$$
\left.\tau^{p,-}\right|_{H}=\int_{-\infty}^{0} \pi^{\lambda} d \lambda
$$

Let $\chi$ be any nontrivial character of $H$. Let $\tau$ be the unitary representation of $P$ induced by $\chi$. By the Mackey subgroup theorem and the triviality of $\chi$ on the center of $H, \tau$ is trivial on the center of $\bar{N}$. Applying Mackey's little subgroup method one obtains

Proposition 4.1. The representations $\tau, \tau^{p, \pm}, p$ an integer, form a complete list of the infinite-dimensional irreducible unitary representations of $P$.

We remark that the finite-dimensional irreducible unitary representations of $P$ are just the characters of $M A$ extended to $P$.

The irreducible representations of $P$ which are nontrivial on the center of $\bar{N}$ play a significant role in the remainder of this paper. They are sufficient to decompose the representations of $\operatorname{SU}(2,1)$ which we construct in the next section.

Let $\alpha$ be a real number and let $c>0$. Let $l_{2}$ be the Hilbert space of all square summable complex sequences $\left(c_{0}, c_{1}, c_{2}, \ldots\right)$. Then

$$
\begin{aligned}
& \mathscr{H}^{+}=L^{2}\left((0, \infty), l_{2}, c|\lambda|^{-\alpha} d \lambda\right), \\
& \mathscr{H}^{-}=L^{2}\left((-\infty, 0), l_{2}, c|\lambda|^{-\alpha} d \lambda\right)
\end{aligned}
$$


are Hilbert spaces. Define representations $R^{ \pm}$on $\mathscr{H}^{\mp}$ by

$$
\begin{aligned}
R^{ \pm}(a(r)) f(\lambda) & =r^{\alpha-1} f\left(\lambda / r^{2}\right), \\
\left(R^{ \pm}(u)\right) f(\lambda)_{m} & =u^{p \mp 3 m} f(\lambda)_{m}, \text { and } \\
\left(R^{ \pm}(h) f\right)(\lambda)_{m} & =\sum_{l}\left\langle\pi^{\lambda}(h)^{-1} e_{m}, e_{l}\right\rangle f(\lambda)_{l} .
\end{aligned}
$$

Clearly $R^{ \pm}(a(r))$ and $R^{ \pm}(u)$ are unitary. Moreover, by Parseval's identity, $\left\|R^{+}(z, t) f(\lambda)\right\|^{2}=\|f(\lambda)\|^{2}$. Thus, $R^{ \pm}(z, t)$ is unitary.

Theorem 4.2. $R^{ \pm}$is a unitary representation of $P$ unitarily equivalent to $\tau^{p, \pm}$. Proof. We show the equivalence of $R^{-}$with $\tau^{p,-}$. Define a linear transformation $W$ from $L^{2}((-\infty, 0), \overline{\mathscr{H}})$ into $\mathscr{H}^{+}$by

$$
(W f)(\lambda)_{m}=c^{-1 / 2}|\lambda|^{\alpha / 2}\left\langle\overline{\left.f(-\lambda), e_{m}\right\rangle} .\right.
$$

Then

$$
\|W f(\lambda)\|^{2}=c^{-1}|\lambda|^{\alpha}\|f(-\lambda)\|^{2}
$$

Hence by (1.8), $W$ is an isometry. Moreover, $W$ is surjective. An easy calculation shows $W \tau^{p,-}(a(r))=R^{-}(a(r)) W$. Furthermore, if $|u|=1$, then

$$
\begin{aligned}
\left(W \tau^{p,-}(u) f\right)(\lambda)_{m} & =c^{-1 / 2}|\lambda|^{\alpha / 2}\left\langle\tau^{p,-}(u) f(-\lambda), e_{m}\right\rangle^{-} \\
& =c^{-1 / 2}|\lambda|^{\alpha / 2}\left\langle u^{p} T_{u} f(-\lambda), e_{m}\right\rangle^{-} \\
& =c^{-1 / 2}|\lambda|^{\alpha / 2} u^{p}\left\langle f(-\lambda), T_{u}^{-1} e_{m}\right\rangle^{-} .
\end{aligned}
$$

But by (4.1), $T_{u}^{-1} e_{m}=u^{-3 m} e_{m}$ on the conjugate space $\overline{\mathscr{H}}$. Thus

$$
\begin{aligned}
W \tau^{p,-}(u) f(\lambda)_{m} & =c^{-1 / 2}|\lambda|^{\alpha / 2} u^{p+3 m} \overline{\left\langle f(-\lambda), e_{m}\right\rangle} \\
& =u^{p+3 m} W f(\lambda)_{m}=R^{-}(u) W f(\lambda)_{m} .
\end{aligned}
$$

Finally note that

$$
\begin{aligned}
W \tau^{p,-}(h) F(\lambda)_{m} & =c^{-1 / 2}|\lambda|^{\alpha / 2}\left\langle\tau^{p,-}(h) f(-\lambda), e_{m}\right\rangle^{-} \\
& =c^{-1 / 2}|\lambda|^{\alpha / 2}\left\langle\pi^{-\lambda}(h) f(-\lambda), e_{m}\right\rangle^{-} \\
& =c^{-1 / 2}|\lambda|^{\alpha / 2}\left\langle f(-\lambda), \pi^{-\lambda}\left(h^{-1}\right) e_{m}\right\rangle^{-} \\
& =c^{-1 / 2}|\lambda|^{\alpha / 2} \sum_{k}\left\langle\pi^{\lambda}\left(h^{-1}\right) e_{m}, e_{k}\right\rangle\left\langle f(-\lambda), e_{k}\right\rangle^{-},
\end{aligned}
$$

since $\left\langle\pi^{-\lambda}\left(h^{-1}\right) e_{m}, e_{k}\right\rangle^{-}=\left\langle\pi^{\lambda}\left(h^{-1}\right) e_{m}, e_{k}\right\rangle$ for $\pi^{\lambda}$ and $\pi^{-\lambda}$ are conjugate representations. Therefore $W \tau^{p,-}(h)=R^{-}(h) W$.

That $R^{+}$is equivalent to $\tau^{p,+}$ is proved similarly.

\section{NULL SPACE REPRESENTATIONS}

The intertwining operators $A(p, \alpha), \operatorname{Re} \alpha>0$ are strongly analytic in the parameter $\alpha$ as operators on $L^{2}(K \backslash M)=\mathscr{H}^{p, \alpha}$. In this section a sequence 
$\mathscr{H}^{p, \alpha_{0}}=N_{0} \supseteq N_{1} \supseteq N_{2} \supseteq \cdots \supseteq N_{k} \supseteq N_{k+1}=0$ of invariant subspaces will be constructed along with invariant nondegenerate forms on the subquotient spaces $N_{i} / N_{i+1}$ for the representation $R^{p, \alpha_{0}}$ at any value $\alpha_{0}$ where $A\left(p, \alpha_{0}\right)$ has nontrivial null space. For expository reasons it is advantageous to develop the setup in the situation where the operators $A(p, \alpha)$ act on the fixed Hilbert space $L^{2}(K / M)$. The natural Hermitian pairing (I.6.6) between $\mathscr{H}^{p,-\alpha}$ and $\mathscr{H}^{p, \alpha}$ is then

$$
\langle f, h\rangle=\int f(k) \overline{h(k)} d k
$$

By (2.9) one has

$$
\left\langle R^{p,-\alpha}(g), f, R^{p, \alpha}(g) h\right\rangle=\langle f, h\rangle \text { for all } g .
$$

Moreover, since $A(p, \alpha)$ intertwines $R^{p, \alpha}$ with $R^{p,-\alpha}$ and by (2.9) the form $\langle$,$\rangle is invariant under R^{p,-\alpha} \times R^{p, \alpha}$, the form $\langle A(p, \alpha) f, h\rangle$ is invariant under the representation $R^{p, \alpha}$.

Suppose now that one has a bounded operator valued strongly analytic function $\alpha \mapsto B(\alpha)$ all defined on a closed subspace $N_{k}$ of $L^{2}(K / m)$ having values in $L^{2}(K / M)$. Furthermore, suppose $N_{k}$ is invariant under the representation $R^{p, \alpha_{0}}$ and one has

$$
B(\alpha) R^{p, \alpha}(g) f=R^{p,-\alpha}(g) B(\alpha) f \text { for all } f \in N_{k}, g \in G .
$$

Define $N_{k+1}=\left\{f \in N_{k}: B\left(\alpha_{0}\right) f=0\right\}$. Then $N_{k+1}$ is a closed subspace of $N_{k}$; and by (5.3) and the invariance of $N_{k}$ under $R^{p, \alpha_{0}}$, it is an invariant subspace of $R^{p, \alpha_{0}}$.

Since $B\left(\alpha_{0}\right)$ intertwines $R^{p, \alpha_{0}}$ and $R^{p,-\alpha_{0}}$ on $N_{k}$, the form $\left\langle B\left(\alpha_{0}\right) f, h\right\rangle$ is invariant under the representation $R^{p, \alpha_{0}}$ and factors to an invariant form on $N_{k} / N_{k+1}$. Define $C(\alpha)$ on $N_{k+1}$ by:

$$
C(\alpha) f= \begin{cases}\frac{B(\alpha)}{\alpha-\alpha_{0}} f & \text { if } \alpha \neq \alpha_{0}, \\ \lim _{\alpha \rightarrow \alpha_{0}} \frac{B(\alpha)}{\alpha-\alpha_{0}} f & \text { if } \alpha=\alpha_{0} .\end{cases}
$$

Then $C(\alpha)$ is a strongly analytic bounded operator valued function defined on $N_{k+1}$ which satisfies (5.3). This procedure can be inductively repeated and we obtain the following result.

Theorem 5.1. Suppose $\alpha_{0}>0$. Then there is a finite sequence

$$
\mathscr{H}^{p, \alpha_{0}}=N_{0} \supseteq N_{1} \supseteq N_{2} \supseteq \cdots \supseteq N_{k} \supseteq N_{k+1}=\{0\}
$$

of closed invariant subspaces for the representation $R^{p, \alpha_{0}}$ such that

$$
\lim _{\alpha \rightarrow \alpha_{0}}\left\langle\frac{A(p, \alpha)}{\left(\alpha-\alpha_{0}\right)^{j}} f, h\right\rangle
$$


defines an invariant form for the subquotient representation of $R^{p, \alpha_{0}}$ on $N_{j} / N_{j+1}$.

The sequence terminates since the principal series have finite Jordan-Hölder series. For later reference we also note:

$$
N_{j+1}=\left\{f: \lim _{\alpha \rightarrow \alpha_{0}} \frac{A(p, \alpha)}{\left(\alpha-\alpha_{0}\right)^{j}} f=0\right\} .
$$

Next we will develop explicit information in regards to the sequence $N_{0} \supseteq$ $N_{1} \supseteq N_{2} \supseteq \cdots \supseteq N_{k} \supseteq N_{k+1}=0$ described in Theorem 5.1. To emphasize the dependence of the distribution $U$ on $\alpha$, we shall sometimes write $U_{a}$.

First one needs to determine when there are $f$ in $\mathscr{H}\left(\alpha_{0}\right)$ satisfying the equality $A\left(p, \alpha_{0}\right) f=0$. By (3.28) one has $\left(A\left(p, \alpha_{0}\right) f\right)_{m, n}=\widehat{f}_{m, n} \widehat{U}_{n, n}$. Thus $A\left(p, \alpha_{0}\right) f=0$ implies $\widehat{f}_{m, n} \widehat{U}_{n, n}=0$ for all $n$. By (b) of Proposition 3.4 and by (3.26) one sees $\widehat{f}_{m, n} \widehat{U}_{n, n}=0$ iff $\widehat{f}_{m, n}=0$, or $c_{n}^{+}\left(\alpha_{0}\right)=0$, or $c_{n}^{-}\left(\alpha_{0}\right)=0$. Indeed if one sets

$$
\begin{aligned}
& \widehat{f}_{m, n}^{+}(\lambda)=1_{(0, \infty)}(\lambda) \widehat{f}_{m, n}(\lambda), \\
& \hat{f}_{m, n}^{-}(\lambda)=1_{(-\infty, 0)}(\lambda) \hat{f}_{m, n}(\lambda),
\end{aligned}
$$

then $\widehat{f}_{m, n} \widehat{U}_{n, n}=0$ occurs if

$$
\begin{array}{lll}
c_{n}^{+}\left(\alpha_{0}\right)=c_{n}^{-}\left(\alpha_{0}\right)=0, & \text { or } & \\
c_{n}^{+}\left(\alpha_{0}\right)=0, & \hat{f}_{m, n}^{-}=0, & \text { or } \\
c_{n}^{-}\left(\alpha_{0}\right)=0, & \hat{f}_{m, n}^{+}=0 . &
\end{array}
$$

Since $\widehat{f}_{m, n}=0$ for all $m, n$ implies $f=0$, one has

Proposition 5.2. The null space $N_{1}$ is nontrivial if and only if there is an $n$ such that either $c_{n}^{+}\left(\alpha_{0}\right)=0$ or $c_{n}^{-}\left(\alpha_{0}\right)=0$. Moreover, if $f \in N_{1}$, then

$$
\begin{aligned}
& \hat{f}_{m, n}^{+}=0 \quad \text { if } c_{n}^{+}\left(\alpha_{0}\right) \neq 0, \\
& \widehat{f}_{m, n}^{-}=0 \quad \text { if } c_{n}^{-}\left(\alpha_{0}\right) \neq 0 \text {. }
\end{aligned}
$$

By (3.24) one obtains

Corollary 5.3. $N_{1} \neq 0$ iff $p+\alpha_{0}$ or $p-\alpha_{0}$ is a nonzero even integer.

Note in [9] Kraljevic showed $R^{p, \alpha_{0}}$ is reducible precisely in these cases.

By (5.4) and (5.5) one sees the operator $A^{j}\left(p, \alpha_{0}\right)$ defined on $N^{j}$ by

$$
A^{j}\left(p, \alpha_{0}\right) f=\lim _{\alpha \rightarrow \alpha_{0}} \frac{A(p, \alpha)}{\left(\alpha-\alpha_{0}\right)^{j}} f
$$


exists in $\mathscr{H}\left(-\alpha_{0}\right)$. In particular, by (3.28) one has

$$
\left(A^{j}\left(p, \alpha_{0}\right) f\right)_{m, n}^{-}=\lim _{\alpha \rightarrow \alpha_{0}}\left(\frac{A(p, \alpha)}{\left(\alpha-\alpha_{0}\right)^{j}} f\right)_{m, n}^{-}=\lim _{\alpha \rightarrow \alpha_{0}} \frac{\widehat{f}_{m, n} \widehat{U}_{n, n}}{\left(\alpha-\alpha_{0}\right)^{j}} .
$$

But by (3.25),

$$
\lim _{\alpha \rightarrow \alpha_{0}} \frac{\widehat{U}_{n, n}}{\left(\alpha-\alpha_{0}\right)^{j}}
$$

is a distribution on $\mathscr{S}_{0}(\mathbb{R})$ defined by the function

$$
\lim _{\alpha \rightarrow \alpha_{0}} \frac{c_{n}^{-}(\alpha)}{\left(\alpha-\alpha_{0}\right)^{j}}|\lambda|^{-\alpha_{0}} 1_{(-\infty, 0)}(\lambda)+\lim _{\alpha \rightarrow \alpha_{0}} \frac{c_{n}^{+}(\alpha)}{\left(\alpha-\alpha_{0}\right)^{j}}|\lambda|^{-\alpha_{0}} 1_{(0, \infty)}(\lambda) .
$$

Define $c_{n, j}^{ \pm}\left(\alpha_{0}\right)$ by

$$
c_{n, j}^{ \pm}\left(\alpha_{0}\right)=\lim _{\alpha \rightarrow \alpha_{0}} \frac{c_{n}^{ \pm}(\alpha)}{\left(\alpha-\alpha_{0}\right)^{j}},
$$

where they are taken to be $\infty$ if the limit does not exist. In summary one has Proposition 5.4. The jth null space $N_{j}$ is nontrivial iff $c_{n, j}^{+}\left(\alpha_{0}\right) \neq \infty$ or $c_{n, j}^{-}\left(\alpha_{0}\right)$ $\neq \infty$ for some $n$. Moreover, if $f \in N_{j}$, then $\widehat{f}_{m, n}^{+}=0$ whenever $c_{n, j}^{+}\left(\alpha_{0}\right)=\infty$ and $\widehat{f}_{m, n}^{-}=0$ whenever $\bar{c}_{n, j}^{-}\left(\alpha_{0}\right)=\infty$.

The above discussion shows that

$$
\left(A^{j}\left(p, \alpha_{0}\right)\right)_{m, n}^{-}=\widehat{f}_{m, n} U_{n, n}^{j},
$$

where $\widehat{U}_{n, n}^{j}$ is the distribution defined by the function

$$
\widehat{u}_{n, n}^{j}(\lambda)= \begin{cases}c_{n, j}^{+}\left(\alpha_{0}\right) /|\lambda|^{\alpha} & \text { if } \lambda>0, \\ c_{n, j}^{-}\left(\alpha_{0}\right) /|\lambda|^{\alpha} & \text { if } \lambda<0 .\end{cases}
$$

By Corollary 5.3 we may assume $\alpha_{0}=p+2 k>0$ for some integer $k$, for otherwise $N_{1}=0$. Then by (5.8) and (3.24)

$$
\begin{gathered}
c_{n, j}^{+}\left(\alpha_{0}\right)=2^{1-j} \pi^{2} \Gamma\left(\alpha_{0}\right) \lim _{x \rightarrow k} \frac{\Gamma(1+n-x)}{(x-k)^{j} \Gamma(1-x) \Gamma(1-p-x) \Gamma(x+p+n+1)}, \\
\text { (5.12) } c_{n, j}^{-}\left(\alpha_{0}\right)=2^{1-j} \pi^{2} \Gamma\left(\alpha_{0}\right) \lim _{x \rightarrow k} \frac{\Gamma(1+n-p-x)}{(x-k)^{j} \Gamma(1-x) \Gamma(1-p-x) \Gamma(x+n+1)} .
\end{gathered}
$$

But the gamma function satisfies

$$
\lim _{x \rightarrow k}(x-k) \Gamma(x-l)=\frac{(-1)^{l-k}}{(l-k) !} \quad \text { if } l \geq k,
$$


and

$$
\lim _{x \rightarrow k}(x-k) \Gamma(l-x)=\frac{(-1)^{l-k+1}}{(k-l) !} \text { if } l \leq k
$$

Hence

$$
\lim _{x \rightarrow k} \frac{\Gamma(l-x)}{\Gamma(m-x)}=(-1)^{l-m} \frac{(k-m) !}{(k-l) !}, \quad k \geq l \text { and } k \geq m
$$

and

$$
\lim _{x \rightarrow k} \frac{\Gamma(l-x)}{\Gamma(x-m)}=(-1)^{l+m-1} \frac{(m-k) !}{(k-l) !}, \quad k \geq l \text { and } k \leq m .
$$

Thus by (5.8)

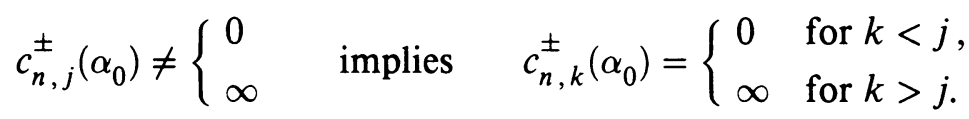

It follows by (5.11) and (5.12) that $c_{n, 3}^{ \pm}\left(\alpha_{0}\right)=\infty$ for all $n$. Thus by Proposition 2.4 , the null space $N_{3}$ is trivial. We thus need only calculate the nonzero, noninfinity values of $c_{n, 0}^{ \pm}\left(\alpha_{0}\right), c_{n, 1}^{ \pm}\left(\alpha_{0}\right)$, and $c_{n, 2}^{ \pm}\left(\alpha_{0}\right)$.

Using (5.11) and (5.12) and the limit properties (5.13)-(5.16) of the gamma function for $\alpha_{0}=p+2 k>0$ one obtains

$$
\begin{aligned}
& c_{n, 0}^{+}\left(\alpha_{0}\right)=(-1)^{n} 2 \pi^{2} \Gamma\left(\alpha_{0}\right) \frac{(k-1) !}{(-k-p) !(k+p+n) !(k-n-1) !} \\
& \text { if } 0 \leq-k-p \leq n<k \text {; } \\
& \left((-1)^{n+p+k} \pi^{2} \Gamma\left(\alpha_{0}\right) \frac{(k-1) !(k+p-1) !}{(k-n-1) !(k+p+n) !}\right. \\
& \text { if } 1 \leq k \text { and } 1 \leq p+k, n<k \text {; } \\
& (-1)^{k-p-1} \pi^{2} \Gamma\left(\alpha_{0}\right) \frac{(-p-n-k-1) !(k-1) !}{(-k-p) !(k-n-1) !} \\
& c_{n, 2}^{+}\left(\alpha_{0}\right)=\left\{\begin{array}{c}
(-1)^{p} \pi^{2} \Gamma\left(\alpha_{0}\right) \frac{(n-k) !(k-1) !(k+p-1) !}{2(k+p+n) !} \\
\text { if } 1 \leq k \leq n \text { and } 1 \leq p+k \\
(-1)^{-p-n-1} \pi^{2} \Gamma\left(\alpha_{0}\right) \frac{(n-k) !(-k-p-n-1) !(k-1) !}{2(-k-p) !} \\
\text { if } 1 \leq k \leq n \text { and } n<-k-p
\end{array}\right.
\end{aligned}
$$




$$
\begin{gathered}
c_{n, 0}^{-}\left(\alpha_{0}\right)=2(-1)^{n} \pi^{2} \Gamma\left(\alpha_{0}\right) \frac{(k+p-1) !}{(-k) !(k+p-n-1) !(n+k) !} \\
\text { if } 0 \leq-k \leq n<k+p ; \\
c_{n, 1}^{-}\left(\alpha_{0}\right)=\left\{\begin{array}{c}
(-1)^{n+k} \pi^{2} \Gamma\left(\alpha_{0}\right) \frac{(k-1) !(k+p-1) !}{(k+p-n-1) !(k+n) !} \\
\text { if } 1 \leq p+k, 1 \leq k, \text { and } n<p+k ; \\
(-1)^{k} \pi^{2} \Gamma\left(\alpha_{0}\right) \frac{(n-p-k) !(k-1) !}{(-k-p) !(k+n) !} \\
\text { if } 0 \leq p+k \text { and } 1 \leq k ; \\
(-1)^{k+1} \pi^{2} \Gamma\left(\alpha_{0}\right) \frac{(-k-n-1) !(k+p-1) !}{(-k) !(k+p-n-1) !} \\
\text { if } 1 \leq k+p, 0 \leq k, \text { and } n<k+p, n<-k ; \\
(-1)^{k+p} \pi^{2} \Gamma\left(\alpha_{0}\right) \frac{(n-p-k) !(k+p-1) !}{(-k) !(n+k) !} \\
\text { if } 1 \leq k+p \leq n \text { and } 0 \leq-k \leq n ; \\
c_{n, 2}^{-}\left(\alpha_{0}\right)=\left\{\begin{array}{c}
(-1)^{p} \pi^{2} \Gamma\left(\alpha_{0}\right) \frac{(n-p-k) !(k-1) !(k+p-1) !}{2(k+n) !} \\
\text { if } 1 \leq p+k \leq n \text { and } 1 \leq k ;
\end{array}\right. \\
(-1)^{n+p+1} \pi^{2} \Gamma\left(\alpha_{0}\right) \frac{(n-k-p) !(k+p-1) !(-n-k-1) !}{2(-k) !} \\
\text { if } 1 \leq k+p \leq n \text { and } n<-k
\end{array}\right.
\end{gathered}
$$

The formulas (5.18)-(5.23) along with (5.17) and Proposition 5.4 determine when the spaces $N_{0}, N_{1}$, and $N_{2}$ are nontrivial.

In the remaining part of this section we will determine explicit expressions for the invariant forms

$$
\left\langle A^{j}\left(p, \alpha_{0}\right) f, h\right\rangle \text { on } N_{j} / N_{j+1},
$$

in the cases $j=1$ and $j=2$. The $j=0$ case depends on the distribution $U_{\alpha_{0}}$ on the singular set $\mathbf{C} \times\{0\}$ for which the methods developed in Part I are not applicable.

In order to facilitate the calculations of these forms it will be advantageous to introduce the $R^{p, \alpha}$ invariant subspaces $\mathscr{H}_{\infty}^{p, \alpha}$ consisting of the $C^{\infty}$ functions in $\mathscr{Z}^{p, \alpha}$. It is known that the space $\mathscr{H}_{\infty}^{p, \alpha}$ contains the $K$-finite vectors for the representation $R^{p, \alpha}$ and that any closed invariant subspace for $R^{p, \alpha}$ contains a dense subspace of $K$ finite vectors. Hence if $N_{j}^{\infty}=N_{j} \cap \mathscr{H}_{\infty}^{p, \alpha}$, then $N_{j}^{\infty}$ is a dense invariant subspace of $N_{j}$; and if $\mathscr{H}_{K}^{p, \alpha}$ is the space of $K$ finite vectors in $\mathscr{H}^{p, \alpha}$, then $N_{i, K}=N_{j} \cap \mathscr{H}_{K}^{p, \alpha}$ is a dense but not invariant subspace of $N_{j}^{\infty}$.

The main reason for introducing $\mathscr{H}_{\infty}^{p, \alpha}$ is the following fact which is a consequence of Lemma 8.10.13 in [20] or an explicit calculation using the Iwasawa 
decomposition of $G$. If $f \in \mathscr{H}_{\infty}^{p, \alpha}$, then $f$ is $C^{\infty}$ on $H$ and

$$
\lim _{(z, t) \rightarrow \infty}\left(\left(1+|z| / 2^{2}\right)^{2}+t^{2}\right)^{\alpha / 2+1} f(z, t) \text { exists. }
$$

By (5.24) it follows that $f$ is a bounded $L^{1}$ function when $f \in \mathscr{H}_{\infty}^{p, \alpha}$ and $\alpha>0$. Also note by (I.5.5) and (3.4) that

$$
\begin{aligned}
\langle A(p, \alpha) f, h\rangle=\langle U * f, h\rangle & \\
\quad & \iint U * f(z, t) \overline{h(z, t)} d z d t \\
& =\iiint \int f\left((w, s)^{-1}(z, t)\right) \bar{h}(z, t) d z d t U(w, s) d w d s \\
& =\iiint \int f((w, s)(-z,-t)) h^{*}(z, t) d z d t U(-w,-s) d w d s \\
& =\check{U}\left(f * h^{*}\right) .
\end{aligned}
$$

But $f * h^{*}$ is a bounded $L^{1}$ function if $f$ and $h$ are in $\mathscr{H}_{\infty}^{p, \alpha}$. Thus by (3.6), Theorem I.5.2, and the remarks following it and the fact that $\widehat{U}_{m, n}=0$ when $m \neq n$ one obtains

Theorem 5.5. Suppose $0<\alpha<1$ and $f, h \in \mathscr{H}_{\infty}^{p, \alpha}$. Then

$$
\begin{gathered}
\langle A(p, \alpha) f, h\rangle=\frac{1}{2 \pi^{2}} \sum_{m} \int|\lambda| \widehat{u}_{m, m}(\lambda)\left(f * h^{*}\right)_{m, m}(\lambda) d \lambda \\
=\frac{1}{2 \pi^{2}}\left(\int_{-\infty}^{0}|\lambda|^{1-\alpha} \operatorname{tr}\left(\pi^{\lambda}\left(f * h^{*}\right) \bar{\pi}(U)\right) d \lambda\right. \\
\left.\quad+\int_{0}^{\infty}|\lambda|^{1-\alpha} \operatorname{tr}\left(\pi^{\lambda}\left(f * h^{*}\right) \pi(U)\right) d \lambda\right) .
\end{gathered}
$$

Since $\bar{\pi}(U)$ and $\pi(U)$ are diagonal relative to the basis $e_{n}$ with eigenvalues $c_{n}^{-}(\alpha)$ and $c_{n}^{+}(\alpha)$, the formula (3.24) would determine when the representation $R^{p, \alpha}$ is in the complementary series for $0<\alpha<1$. This occurs precisely when all the constants $c_{n}^{ \pm}(\alpha)$ are both all positive or both all negative.

Next we determine the form $\left\langle A^{j}(p, \alpha) f, h\right\rangle$ on the subspace $N^{j} \cap\{f: \hat{f} \in$ $\left.\mathscr{S}_{0}(H)\right\}$. Note by (5.9) that $\left(A^{j}\left(p, \alpha_{0}\right) f\right)_{m, n}=\widehat{f}_{m, n} \widehat{U}_{n, n}^{j}$ where $\widehat{U}_{n, n}^{j}$ is the distribution defined in (5.10). Recall by Proposition 5.4 that if $f \in N_{j}$, then $\widehat{f}_{m, n}^{+}=0$ if $c_{n, j}^{+}\left(\alpha_{0}\right)=\infty$, and $\widehat{f}_{m, n}^{-}=0$ if $c_{n, j}^{-}\left(\alpha_{0}\right)=\infty$. Note by (I.5.2) that for any distribution $U$, one has

$$
\begin{aligned}
(\check{U})_{m, n}^{\frown}(\phi) & =\int d \check{U}(z, t) \int \phi(\lambda) e^{-i \lambda t} h_{m, n}(z, \lambda) d \lambda \\
& =\int d U(z, t) \int \phi(\lambda) e^{i \lambda t} h_{m, n}(-z, \lambda) d \lambda \\
& =\int d U(z, t) \int \phi(-\lambda) e^{-i \lambda t} h_{m, n}(-z,-\lambda) d \lambda
\end{aligned}
$$


But by (I.5.3)

$$
\begin{aligned}
h_{m, n}(-z,-\lambda) & =\left\langle\pi^{-\lambda}(-z, 0) e_{m}, e_{n}\right\rangle \\
& =\left\langle\pi^{-\lambda}(z, 0) e_{n}, e_{m}\right\rangle^{-}=\left\langle\pi^{\lambda}(z, 0) e_{n}, e_{m}\right\rangle,
\end{aligned}
$$

since $\pi^{\lambda}$ and $\pi^{-\lambda}$ are conjugate representations. Thus

$$
(\check{U})_{m, n}(\phi)=\int d U(z, t) \int \phi(-\lambda) e^{-i \lambda t} h_{n, m}(z, \lambda) d \lambda=\int d \widehat{U}_{n, m}(\lambda) \phi(-\lambda) .
$$

Hence

$$
(\check{U})_{m, n}=\left(\widehat{U}_{n, m}\right)^{\vee}
$$

Similarly,

$$
(\bar{f})_{m, n}(\lambda)=\overline{\bar{f}_{m, n}(-\lambda)}
$$

Thus by Theorem I.5.1 and by (5.9), (5.25), and (5.26)

$$
\begin{aligned}
\left\langle A^{j}\left(p, \alpha_{0}\right) f, h\right\rangle & =\left(\left(A^{j}\left(p, \alpha_{0}\right) f\right)^{\vee}\right)^{\vee}(\bar{h}) \\
& =\frac{1}{2 \pi^{2}} \sum_{k, m}\left(\left(A^{j}\left(p, \alpha_{0}\right) f\right)^{\vee}\right)_{k, m}\left(|\lambda|(\bar{h})_{m, k}(\lambda)\right) \\
& =\frac{1}{2 \pi^{2}} \sum_{k, m}\left(A^{j}\left(p, \alpha_{0}\right) f\right)_{m, k} \vee \overline{\left(|\lambda| \widehat{h}_{m, k}(-\lambda)\right)} \\
& =\frac{1}{2 \pi^{2}} \sum_{k, m}\left(A^{j}\left(p, \alpha_{0}\right) f\right)_{m, k}\left(|\lambda| \widehat{\widehat{h}}_{m, k}(\lambda)\right) \\
& =\frac{1}{2 \pi^{2}} \sum_{k, m} \widehat{f}_{m, k} \widehat{U}_{k, k}^{j}\left(|\lambda| \widehat{\widehat{h}}_{m, k}(\lambda)\right) .
\end{aligned}
$$

Using the convention $0 \cdot \infty=0$, Proposition 5.4 and (5.10) then imply the following theorem.

Theorem 5.6. Suppose $f, h \in N_{j}$ and $\hat{f}, \widehat{h}$ vanish for $\lambda$ near 0 . Then

$$
\begin{array}{r}
\left\langle A^{j}\left(p, \alpha_{0}\right) f, h\right\rangle=\frac{1}{2 \pi^{2}}\left(\sum_{m, k} \int_{-\infty}^{0} \frac{c_{k, j}^{-}\left(\alpha_{0}\right)}{|\lambda|^{\alpha_{0}-1}} \widehat{f}_{m, k}(\lambda) \overline{\widehat{h}_{m, k}(\lambda)} d \lambda\right. \\
\left.\quad+\int_{0}^{\infty} \frac{c_{k, j}^{+}\left(\alpha_{0}\right)}{|\lambda|^{\alpha_{0}-1}} \widehat{f}_{m, k}(\lambda) \widehat{\widehat{h}_{m, k}(\lambda)} d \lambda\right) .
\end{array}
$$

We remark that if $c_{k, j}^{ \pm}\left(\alpha_{0}\right)=\infty$, then $\widehat{f}_{m, k}(\lambda)=0$ for $\pm \lambda>0$.

\section{UNITARIZABLE SUBQUOTIENTS}

In this section we determine $\alpha_{0}$ and $p$ for which the subquotient $N_{1} / N_{2}$ or $N_{2}$ is unitary. This can be determined using Theorem 5.2, Proposition 5.4, and the formulas (5.18) to (5.23). We next look at the representation $R^{p, \alpha_{0}}$ on the subquotient $N_{j} / N_{j+1}$. 
Theorem 6.1. The representation $R^{p, \alpha_{0}}$ is unitary on $N_{j} / N_{j+1}$ iff those $c_{n, j}^{ \pm}\left(\alpha_{0}\right)$ which are nonzero and noninfinity are all positive or all negative. Moreover, the representation $R^{p, \alpha_{0}}$ extended to the completion of $N_{j} / N_{j+1}$ is equivalent on subgroup $M A \bar{N}$ to the representation

$$
\sum_{c_{n, j}^{-}\left(\alpha_{0}\right) \notin\{0, \infty\}} \tau^{p-3 n,+} \oplus \sum_{c_{n, j}^{+}\left(\alpha_{0}\right) \notin\{0, \infty\}} \tau^{p+3 n,-} .
$$

Proof. The first statement is a consequence of Theorem 5.2 and Proposition 5.4. To see the second we use Theorem 4.2. Indeed, fix $k$ and + or - where $c_{n, j}^{ \pm}\left(\alpha_{0}\right) \notin\{0, \infty\}$. Then using (2.7), (I.1.8), (I.3.17), and (I.5.3) note that

$$
\begin{aligned}
\left(R^{\left.p, \alpha_{0}\right)}(a(r)) f\right)_{m, n}(\lambda) & =\int R^{p, \alpha_{0}}(a(r)) f(z, t)\left\langle\pi^{\lambda}(z, t) e_{m}, e_{n}\right\rangle d z d t \\
& =r^{\alpha_{0}+2} \int f\left(r z, r^{2} t\right)\left\langle\pi^{\lambda}(z, t) e_{m}, e_{n}\right\rangle d z d t \\
& =r^{\alpha_{0}-2} \int f(z, t)\left\langle\pi^{\lambda / r^{2}}(z, t) e_{m}, e_{n}\right\rangle d z d t .
\end{aligned}
$$

Hence

$$
\left(R^{p, \alpha_{0}}(a(r)) f\right)_{m, n}^{\sim}(\lambda)=r^{\alpha_{0}-2} \widehat{f}_{m, n}\left(\lambda / r^{2}\right) .
$$

Next note by (2.8), (4.2), and (I.1.8) that

$$
\begin{aligned}
\left(R^{p, \alpha_{0}}(u) f\right)_{m, n}^{-}(\lambda) & =\int R^{p, \alpha_{0}}(u) f(z, t)\left\langle\pi^{\lambda}(z, t) e_{m}, e_{n}\right\rangle d z d t \\
& =\int u^{p} f\left(u^{-3} z, t\right)\left\langle\pi^{\lambda}(z, t) e_{m}, e_{n}\right\rangle d z d t \\
& =\int u^{p} f(z, t)\left\langle\pi^{\lambda}\left(u^{3} z, t\right) e_{m}, e_{n}\right\rangle d z d t \\
& =\int u^{p} f(z, t)\left\langle\pi^{\lambda}(z, t) T_{u}^{-1} e_{m}, T_{u}^{-1} e_{n}\right\rangle d z d t .
\end{aligned}
$$

But if $\lambda>0$, then (4.1) and the definition of $e_{n}$ implies $T_{u}^{-1} e_{l}=u^{3 l} e_{l}$; while if $\lambda<0$, then since $\pi^{\lambda}$ is defined on $\overline{\mathscr{H}}$ one has $T_{u}^{-1} e_{l}=u^{-3 l} e_{l}$. Thus

$$
\left(R^{p, \alpha_{0}}(u) f\right)_{m, n}^{-}(\lambda)= \begin{cases}u^{p+3(m-n)} \widehat{f}_{m, n}(\lambda) & \text { if } \lambda>0, \\ u^{p-3(m-n)} \widehat{f}_{m, n}(\lambda) & \text { if } \lambda<0 .\end{cases}
$$

Finally (2.6) implies that for $x \in H$, one has

$$
\begin{aligned}
\left(R^{p, \alpha_{0}}(h) f\right)_{m, n}^{-}(\lambda) & =\int_{H} R^{p, \alpha_{0}}(x) f(y)\left\langle\pi^{\lambda}(y) e_{m}, e_{n}\right\rangle d y \\
& =\int_{H} f(y x)\left\langle\pi^{\lambda}(y) e_{m}, e_{n}\right\rangle d y \\
& =\int_{H} f(y)\left\langle\pi^{\lambda}(y) \pi^{\lambda}\left(x^{-1}\right) e_{m}, e_{n}\right\rangle d y \\
& =\int_{H} f(y)\left\langle\pi^{\lambda}\left(x^{-1}\right) e_{m}, e_{l}\right\rangle\left\langle\pi^{\lambda}(y) e_{l}, e_{n}\right\rangle d y .
\end{aligned}
$$


Hence

$$
\left(R^{p, \alpha_{0}}(h) f\right)_{m, n}(\lambda)=\sum_{l}\left\langle\pi^{\lambda}\left(x^{-1}\right) e_{m}, e_{l}\right\rangle \hat{f}_{l, n}(\lambda) .
$$

It follows from Theorem 4.2 and (4.10), (4.11), (4.12), and (6.1), (6.2), and (6.3) that if $c_{n, j}^{-}\left(\alpha_{0}\right) \notin\{0, \infty\}$, then $\tau^{p-3 n,+}$ is a subrepresentation of $\left.R^{p, \alpha_{0}}\right|_{M A \bar{N}}$ having multiplicity one. A similar argument shows that $\tau^{p+3 n,-}$ is a subrepresentation when $c_{n, j}^{+}\left(\alpha_{0}\right) \notin\{0, \infty\}$.

Theorem 6.1 completely describes the representation $R^{p, \alpha_{0}}$ on the minimal parabolic subgroup $M A \bar{N}$ for the quotient space $N_{j} / N_{j+1}$. To determine the spectral decomposition one need only inspect the formulas (5.18) to (5.23). The same argument can be applied to the complementary series.

Theorem 6.2. If $R^{p, \alpha}$ is a complementary series representation, then

$$
\left.R^{p, \alpha}\right|_{M A \bar{N}} \simeq \sum_{n}\left(\tau^{p-3 n,+} \oplus \tau^{p+3 n,-}\right) .
$$

Remark. One can show $R^{p, \alpha}$ is in the complementary series if the $c_{n}^{ \pm}(\alpha)$ are all positive or all negative. By (3.24) this occurs when $p=0$ and $0<\alpha<2$ or when $p= \pm 1$ and $0<\alpha<1$.

We next apply Theorem 6.1 to list all the cases where $R^{p, \alpha_{0}}$ is unitary on $N_{1}$ or on $N_{1} / N_{2}$. This entails an inspection of the coefficients $c_{n, j}^{ \pm}\left(\alpha_{0}\right)$ where $\alpha_{0}=p+2 k$.

Note that when $p+k \geq 1$, and $k \geq 1$, then $(-1)^{p} c_{n, 2}^{ \pm}$are all positive when they are nonzero and noninfinite. Thus

Theorem 6.3. If $\alpha=p+2 k, p+k \geq 1$, and $k \geq 1$, then $R^{p, \alpha}$ is unitary on $N_{2}$ and

$$
\left.R^{p, \alpha}\right|_{M A \bar{N}} \simeq \sum_{n \geq p+k}\left(\tau^{p-3 n,+} \oplus \tau^{p+3 n,-}\right) .
$$

Next note that $c_{n, 1}^{ \pm}(2)$ are all positive. Indeed the only nonzero, noninfinite terms are $c_{1,1}^{ \pm}(2)$. Hence:

Theorem 6.4. The representation $R^{0,2}$ is unitary on $N_{1} / N_{2}$ and on the minimal parabolic subgroup $M A \bar{N}$ is equivalent to

$$
\tau^{-3,+} \oplus \tau^{3,-} .
$$

It follows from Theorems 6.3 and 6.4 that $R^{0,2}$ is unitary on both $N_{1} / N_{2}$ and on $N_{2}$. Kraljevic in [9] has shown that $R^{0,2}$ has four unitarizable irreducible subquotients. One can show that $N_{0} / N_{1}$ gives the trivial representation of $\mathrm{SU}(2,1)$. Hence either the unitary quotient $N_{1} / N_{2}$ or the unitary subspace $\mathrm{N}_{2}$ is reducible.

Next note if $\alpha_{0}=-1$ and $p=-2 k-1$ where $k \geq 1$, then the only nonzero noninfinite coefficient is $c_{k, 2}^{+}(-1)$. If $p=-2 k-1$ where $k \leq-2$, then the only nonzero noninfinite coefficient is $c_{-k-1,2}^{-}(-1)$. 
Theorem 6.5. The representations

$$
R^{ \pm 3,-1}, R^{ \pm 5,-1}, R^{ \pm 7,-1}, \ldots
$$

are unitary on $N_{2}$ and on $M A \bar{N}$ are unitarily equivalent to

$$
\tau^{0, \pm}, \tau^{\mp 1, \pm}, \tau^{\mp 2, \pm}, \ldots .
$$

These are examples of unitary representations of $\operatorname{SU}(2,1)$ which are irreducible on the much smaller minimal parabolic subgroup $P$.

Finally, assume $p$ and $\alpha$ are odd integers with $\alpha=p+2 k$. Then if $k>0$ and $k+p \leq 0$ then $c_{n, 2}^{ \pm}(\alpha)$ are nonzero and noninfinite with the same sign. Moreover, $c_{n, 1}^{-}(\alpha)$ is nonzero noninfinite for all $n$ while $c_{n, 1}^{+}(\alpha)$ are nonzero noninfinite when $n<\frac{\alpha-p}{2}, n<-\frac{\alpha+p}{2}$, or when $n \geq \frac{\alpha-p}{2}, n \geq-\frac{\alpha+p}{2}$. If $k \leq 0$ and $p+k \geq 1$, then again all the coefficients $c_{n, 1}^{ \pm}(\alpha)$ have the same signs and the nonzero noninfinite ones for $c_{n, 1}^{-}(\alpha)$ have parameters $n<-\frac{\alpha-p}{2}, n<\frac{\alpha+p}{2}$, or $n \geq-\frac{\alpha-p}{2}, n \geq \frac{\alpha+p}{2}$ while $c_{n, 1}^{+}(\alpha)$ is nonzero noninfinite for all $n$. Hence we can conclude

Theorem 6.6. Suppose $\alpha$ and $p$ are odd integers. Then if $\alpha+p \leq 0$ and $\alpha-p \geq 0$, or if $\alpha+p \geq 2$ and $\alpha-p \leq 0$, the representation $R^{p, \alpha}$ is unitary on $N_{1} / N_{2}$. Moreover,

$$
\left.R^{p, \alpha}\right|_{M A \bar{N}} \simeq \sum_{n} \tau^{p \mp 3 n, \pm} \oplus \sum_{n<\min \left\{ \pm \frac{\alpha-p}{2}, \mp \frac{\alpha+p}{2}\right\}} \tau^{p \pm 3 n, \mp} \oplus \sum_{n \geq \max \left\{ \pm \frac{\alpha-p}{2}, \frac{\alpha+p}{2}\right\}} \tau^{p \pm 3 n, \mp}
$$

where the upper symbols are used in the first case and the lower symbols are used in the second.

These unitarizable subquotients exhaust most of the irreducible unitary subquotients of the principal representations $R^{p, \alpha}$ for $\alpha>0$. This can be argued using the results of Kraljevic in the cases where there is exactly one unitary subquotient. However, Kraljevic showed there are cases when there are two, three, or four unitary subquotients. Where this occurs is an instance where the subspace $N_{i} / N_{i+1}$ is reducible.

\section{REFERENCES}

1. A. Abramowitz and I. A. Stegun, Handbook of mathematical functions, National Bureau of Standards, Washington, D.C., 1964.

2. L. Corwin and F. P. Greenleaf, Fourier transforms of smooth functions on certain nilpotent Lie groups, J. Funct. Anal. 37 (1980), 203-217.

3. M. Cowling and A. Koranyi, Harmonic analysis on Heisenberg type groups from a geometric viewpoint, Lie Groups Representations. III, Lecture Notes in Math., vol. 1077, Springer, 1984, pp. 60-100.

4. M. Cowling, Harmonic analysis on some nilpotent groups, Topics in Modern Analysis, Vols. I, II, Turin/Milan, 1982, pp. 81-123.

5. R. Fabec, Localizable representations of the De Sitter group, J. Analyse Math. 35 (1979), 151-207. 
6. R. Howe, Quantum mechanics and partial differential equations, J. Funct. Anal. 38 (1980), 188-254.

7. A. W. Knapp and E. M. Stein, Intertwining operators for semisimple groups, Ann. of Math. (2) 93 (1971), 489-578.

8. H. Kraljevic, Representations of the universal covering group $\mathrm{SU}(n, 1)$, Glas. Mat. Ser. III 8 (28) (1973), 23-72.

9. __ On representations of the group $\mathrm{SU}(n, 1)$, Trans. Amer. Math. Soc. 221 (1976), 433-448.

10. R. A. Kunze and E. M. Stein, Uniformly bounded representations and harmonic analysis on the $2 \times 2$ real unimodular group, Amer. J. Math. 82 (1960), 1-62.

11. _ Uniformly bounded representations, II. Analytic continuation of the principal series of representations of the $n \times n$ complex unimodular group, Amer. J. Math. 83 (1961), 723-786.

12. __ Uniformly bounded representations, III. Intertwining operators for the principal series on semisimple groups, Amer. J. Math. 83 (1967), 385-442.

13. R. J. Lipsman, Uniformly bounded representations of the Lorentz groups, Amer. J. Math. 91 (1969), 983-962.

14. G. W. Mackey, Unitary representations of group extensions. I, Acta Math. 99 (1958), 265311.

15. C. Moore and J. Wolf, Square integrable representations of nilpotent Lie groups, Trans. Amer. Math. Soc. 185 (1973), 445-462.

16. P. J. Sally, Jr., Analytic continuation of the irreducible unitary representations of the universal covering group of $\operatorname{SL}(2, \mathbf{R})$, Mem. Amer. Math. Soc. No. 69 (1967), 94 pp.

17. G. Schiffman, Intégrales d'entralacement et fonctions de Whittaker, Bull. Soc. Math. France 99 (1971), 3-72.

18. R. J. Stanke, Analytic uniformly bounded representations of $\mathrm{SU}(1, n+1)$, Trans. Amer. Math. Soc. 290 (1985), 281-302.

19. F. Treves, Topological vector spaces, distributions and kernels, 1st ed., Academic Press, New York, 1967.

20. N. R. Wallach, Harmonic analysis on semisimple Lie groups. I, 1st ed., Dekker, New York, 1973.

21. D. P. Zelobenko, Discrete symmetry operators for reductive Lie groups, Izv. Akad. Nauk SSSR Ser. Mat. 40 (1976), 1055-1083; English transl., Math. USSR-Izv. 10 (1976).

22. __ A description of the quasi-simple irreducible representations of the groups $\mathrm{U}(n, 1)$ and $\operatorname{Spin}(n, 1)$, Izv. Akad. Mat. 41 (1977), 31-50; English transl., Math. USSR Izv. (1977).

Department of Mathematics, Louisiana State Univeristy, Baton Rouge, Louisiana 70803 